\title{
Anti-correlated time lags in the $Z$ source GX 5-1: Possible evidence for a truncated accretion disk
}

\author{
K. Sriram, C. S. Choi \\ Korea Astronomy and Space Science Institute, Daejeon 305-348, Republic of Korea \\ astrosriram@yahoo.co.in
}

A. R. Rao

Tata Institute of Fundamental Research, Mumbai 400005, India

Received — 


\begin{abstract}
We investigate the nature of the inner accretion disk in the neutron star source GX 5-1 by making a detailed study of time lags between X-rays of different energies. Using the cross-correlation analysis, we found anti-correlated hard and soft time lags of the order of a few tens to a few hundred seconds and the corresponding intensity states were mostly the horizontal branch (HB) and upper normal branch (NB). The model independent and dependent spectral analysis showed that during these time lags the structure of accretion disk significantly varied. Both eastern and western approaches were used to unfold the X-ray continuum and systematic changes were observed in soft and hard spectral components. These changes along with a systematic shift in the frequency of quasi-periodic oscillations (QPOs) made it substantially evident that the geometry of the accretion disk is truncated. Simultaneous energy spectral and power density spectral study shows that the production of the horizontal branch oscillations (HBOs) are closely related to the Comptonizing region rather than the disk component in the accretion disk. We found that as the HBO frequency decreases from the hard apex to upper $\mathrm{HB}$, the disk temperature increases along with an increase in the coronal temperature which is in sharp contrast with the changes found in black hole binaries where the decrease in QPO frequency is accompanied by a decrease in the disk temperature and a simultaneous increase in the coronal temperature. We discuss the results in the context of re-condensation of coronal material in the inner region of the disk.
\end{abstract}

Subject headings: accretion, accretion disk—binaries: close—stars: individual (GX 5-1)_X-rays: binaries 


\section{Introduction}

Neutron star low mass X-ray binaries (LMXBs) can be divided into two broad classes viz. Z-type and atoll-type, based on their color-color diagram (CCD) or hardness-intensity diagram (HID), where the Z-type sources have luminosities close to the Eddington limit (van der Klis 2006). It has been suggested that the specific positions on the CCD or HID tracks indicate a different physical configuration of an accretion disk or radiation process (Hasinger \& van der Klis 1989; Hasinger et al. 1990). The Z-type sources traverse a 'Z'-shaped path on HID which consists of three main branches viz. horizontal branch (HB), normal branch (NB) and flaring branch (FB), where NB connects to the $\mathrm{HB}$ and NB.

The Z-type sources can be subdivided into Sco X-1-like (e.g., GX 17+2 and GX 349+2) and Cyg X-2-like (e.g., GX 5-1 and GX 340+0) (Kuulkers et al. 1994). The Sco X-1-like sources exhibit a vertical HB whereas the latter sources exhibit a horizontal HB (Kuulkers et al. 1997). It was suggested that the difference is due either to the magnetic field strength of the neutron star (Psaltis et al. 1995) or to the inclination angle to the line of sight (Hasinger et al. 1989; Hasinger et al. 1990; Kuulkers et al. 1994). However, recent studies on the source XTE J1701-462 ruled out both the possibilities (Homan et al. 2010) because this source showed both Cyg X-2- and Sco X-1-like properties (Homan et al. 2007). The physical parameter which causes the motion along the $\mathrm{Z}$ track on HID was considered to be the mass accretion rate, where the rate increases from the $\mathrm{HB}$ to NB and peaks at the FB. It was also proposed that the accretion rate increases from the FB to HB, which is exactly the opposite case (Jackson et al. 2009). A detailed study on the source XTE J1701-462 suggested that an instability may cause the $\mathrm{Z}$ track rather than the accretion rate because in the study the accretion rate was nearly constant along the tracks (Lin et al. 2009; Homan et al. 2010).

There has been no consensus on the origin of soft X-rays for Z-type sources. The neutron star surface or the Keplerian component of the disk could be the possible site. The hard component 
in the X-ray spectrum is considered to originate from the Comptonization of low energy photons in a hot corona, whose location and structure are not yet properly understood. There are three approaches to model the X-ray spectra of Z-type sources, western, eastern and hybrid model (Lin et al. 2009). The western model (White et al. 1986) consists of a high energy (or power-law) component which is radiating by the Comptonization from the inner region of the disk and a soft/black body component radiating from the surface or close to the surface of neutron star (NS). In the eastern model (Mitsuda et al. 1984), the soft component is assumed to originate from the accretion disk which radiates as a multi-temperature black body and the hard component arises from the Compton cloud/corona assumed to be located at the inner region of the disk or close to the NS surface (Di Salvo et al. 2000, 2001; Agrawal \& Misra 2009). Generally the shape of Compton cloud/corona is considered to be quasi-spherical (Zdziarski et al. 1996; Done et al. 2007) but the studies based on dipping LMXBs show that corona is more likely to be an extended body over the disk (Church \& Balucinska-Church 2001; Jackson et al. 2009). In black hole X-ray binaries (BHXBs), the unfolded spectrum to some extent resembles the eastern model (i.e. soft component from the disk and hard component from the Compton cloud or at the base of jet) and a geometry of truncated accretion disk is often implemented to explain the hard spectral state in BHXBs (see Done et al. 2007 for a review). Both western and eastern models, however, are generally found to be successful in explaining the spectra of neutron star LMXBs (Hasinger et al. 1990) but for some sources hybrid model is preferred over the former models (Lin et al. 2007; $\mathrm{Ng}$ et al. 2010). Generally during an outburst in BHXBs, the spectrum is dominated by the hard component (along with a strong radio emission) and disappears as the disk moves toward the last stable orbit (Remillard \& McClintock 2006; Done et al. 2007). Similar phenomenon is often observed in Z-type sources where the hard X-ray tail and radio emission are observed at HB and fade out when the source is at FB (Di Salvo et al. 2001; Migliari et al. 2007; Lin et al. 2009).

The signature of the Comptonization process can be obtained from cross-spectral studies. Both hard and soft lags of the order of a few micro/milli seconds exist, which are considered to be 
the possible time taken by the soft photons to reprocess as hard photons in the hot corona (van der Klis et al. 1987). Nobili et al. (2000) suggested an inhomogeneous Compton cloud model to explain the soft lags. Such kind of lags were seen in GX 5-1 and the detection of lags were related to the specific position on the Z-track (Vaughan et al. 1999; Qu et al. 2004). Anti-correlated hard or soft lags greater than a few tens of seconds, however, pertains to the pivoting of the spectrum, where anti-correlation means that an intensity variation is opposite between soft and hard X-rays and hard/soft lag means that hard/soft X-ray photons are relatively delayed in their arrivals at the observer. On the basis of a simple Comptonization model, Zdziarski et al. (2002) found that the spectral pivoting (and hence the anti-correlation) in the BHXB Cyg X-1 can be explained by assuming a variable seed photon flux for the Comptonization process. Any delays in such pivoting process will shed light on the physical processes responsible for the generation of the seed photons and/or the re-distribution of geometry of the corona.

Anti-correlated X-ray lags of a few hundred seconds were often seen in some BHXBs (Choudhury et al. 2005; Sriram et al. 2007; Sriram et al. 2009; Sriram et al. 2010) and based on the observed spectral pivoting the lags were explained in the context of truncated accretion disk scenario. The simulation carried out by Sriram et al. (2010) rules out the spurious nature of the observed anti-correlation. So far such anti-correlated hard and soft lags (a few 10-100 s) were detected only for one neutron star source, Cyg X-2, and most of the corresponding observations were located at the HB and upper NB on the Z-track (Lei et al. 2008). Detection of such lags in neutron star LMXBs is important because it indirectly helps to know the radiative and geometrical structure of the accretion disk (which is poorly known in the case of NS LMXBs). The aforementioned arguments are the motivation of our study and we carry out a systematic timing analysis, cross-correlation analysis and the study of power density spectrum (PDS), along with the spectral analysis for the source GX 5-1.

GX 5-1 is the second brightest persistent LMXB located at the Sagittarius region (Bradt et al. 
1968; Fisher et al. 1968) and it is classified as a Z-type source (Hasinger \& van der Klis 1989). The horizontal branch oscillations (HBOs) with frequencies between $13-50 \mathrm{~Hz}$ were discovered by van der Klis et al. (1985) and the normal branch oscillations (NBOs) with frequencies $\sim 6 \mathrm{~Hz}$ were found by Lewin et al. (1992) in GX 5-1. Recently, Sriram et al. (2011b) found that coupled HBO and NBO variations in the source can be interpreted as the physically modified inner disk front. The $\mathrm{kHz}$ quasi-periodic oscillations (QPOs) with frequencies ranging from $200-800 \mathrm{~Hz}$ have been detected at the HB and upper part of NB (Wijnands et al. 1998), in which the QPO peaks are not constantly separated (Jonker et al. 2002). However, no QPOs have been detected at the FB. GX 5-1 shows an inherent hard X-ray tail (power-law index of $\Gamma \sim 1.8$ ) and a Gaussian-like residuals feature which is found at $\sim 10 \mathrm{keV}$ over the eastern and western models (Asai et al. 1994). The existence of jets in GX 5-1 was inferred from the detection of radio emission (Penninx et al. 1989; Tan et al. 1992; Fender \& Hendry 2000). Emission in infrared wavelength was also detected (Jonker et al. 2000).

\section{Data Reduction and Analysis}

We analyzed the public archival data obtained with the Proportional Counter Array (PCA) on board the Rossi-XTE satellite. The PCA consists of five identical proportional counter units which typically cover the energy range of $2-60 \mathrm{keV}$ (Jahoda et al. 2006). Among the available 183 data sets for GX 5-1, we selected 74 data sets that have exposures greater than $2000 \mathrm{~s}$. For spectral analysis, we extracted energy spectra from PCU2 which is the best calibrated among the PCUs and added $0.5 \%$ systematic errors to account for the calibration uncertainties. HEASOFT v6.8 software was used to reduce the raw data of GX 5-1 and unfolded spectra were obtained using the spectral models in XSPEC v12.5.0 (Arnaud 1996).

To obtain the HID, we defined the hardness ratio as the ratio of background subtracted source counts in $8.7-19.7 \mathrm{keV}$ to those of $6.2-8.7 \mathrm{keV}$ and calculated the intensity in a bin 
size of $128 \mathrm{~s}$ (count rate) for the energy range 2.0 - $19.7 \mathrm{keV}$ (Qu et al. 2004). The channels were carefully selected based on the different RXTE gain epochs and the gain-change effect was corrected. For the cross-correlation analysis, the background subtracted light curves with a bin size of $32 \mathrm{~s}$ (standard 2 mode) were extracted in the soft $(2-5 \mathrm{keV})$ and hard energy band (16 - 30 $\mathrm{keV})$. An uninterrupted individual segment of long duration was considered and the crosscor tool implemented in the XRONOS package was used for the analysis (for more details of the program crosscor, see Sriram et al. 2007; Lei et al. 2008; Sriram et al. 2011a). Generally the shape of cross-correlation function (CCF) is found to be complex but the peak of CCF can be fitted by an inverted Gaussian function (Choudhury \& Rao 2004; Sriram et al. 2007; Lei et al. 2008). We therefore fitted the Gaussian function to the CCF data to calculate delay times and errors at a $90 \%$ confidence level $\left(\Delta \chi^{2}=2.71\right)$. We also used the single bit mode data to analyze the power density spectrum (PDS) of a few observations discussed later.

\section{Temporal Analysis Results}

\subsection{Anti-correlated lags in GX 5-1}

We found that most of the observations for GX 5-1 show a positive correlation between the light curves in $2-5 \mathrm{keV}$ and $16-30 \mathrm{keV}$ and a few of them show an ambiguous correlation or a low level of correlation, i.e. a low correlation coefficient (CC). Those are similar to the observations for Cyg X-2 and BHXBs (e.g., see Choudhury \& Rao 2004). Details of the 57 observations are given in Table 1. It was found that the observations located at the HB and hard apex show a positive correlation, a low level of correlation or an anti-correlation without any time lag. For example, in ObsID 30042-01-03-00 a clear anti-correlation at $\sim-0.52$ was observed without lag. We define the low level of correlation as the $\mathrm{CC}$ value of $\leq \pm 0.35$ and find that many observations fall in this category (Table 1). It is also found that during the observations the source was moving/varying toward all the possible directions along the $\mathrm{Z}$ track (i.e. from the upper HB 
to hard apex and vice-versa).

The positive correlations are generally expected and can be understood in the simple scenario of overall intensity variation. We, however, detected strong anti-correlated X-ray lags from the light curves of 17 observations. The representative samples are displayed in Figure 1. We carried out the cross-correlation analysis for each segment of the light curve (we adopted the full light curve in case that the segments showed a similar order of delay for one observation). The cross-correlation functions (CCFs) as a function of delay are plotted in the right panels of Figure 1. The segments in which the lags are found are marked by the letters ' $a$ ' and ' $b$ ' in the light curves. The minima of CCFs, $0.4-0.6$, suggest that the anti-correlations are very strong (the estimated null hypothesis probabilities are $\left.<10^{-6}\right)$. The observations for which the anti-correlated lags were detected in GX 5-1 are listed in Table 2 together with the delay times that range from a few tens to hundred seconds. Among our selected data sets, we found an anti-correlated hard X-ray lag from 13 observations and found an anti-correlated soft X-ray lag from 4 observations (Figure 1 and Table 2). In one observation, both hard and soft X-ray lags were observed. The obtained delay times are similar to those found in Cyg X-2 (Lei et al. 2008) but significantly lower than those in BHXBs (Choudury \& Rao 2004; Choudhury et al. 2005; Sriram et al. 2009).

\subsection{Lags and their locations on HID}

We obtained the HID using the observations made in the period of 1997 - 1998 because our anti-correlated lags were mostly detected during this period. Furthermore, nine observations were made within a span of two months and hence we presumed that the HID would not be affected by any long-term secular variations. The HID shown in Figure 2 clearly displays the Z-shaped track. The locations on the HID are listed in Table 2 along with the hardness ratios for each observation. For the observations beyond the period, i.e., the last four observations in Table 2, we determined their locations based on the PDS analysis. We found that majority of the lags are located at the 
HB and upper NB. There are only a few lags at the soft apex and FB (see Table 2 and Figure 2). In case of the observations that the lags were detected, the source remained at the HB and hard apex for $\sim 19 \mathrm{hrs}$, at the NB for $\sim 14 \mathrm{hrs,} \mathrm{and} \mathrm{at} \mathrm{the} \mathrm{FB} \mathrm{and} \mathrm{soft} \mathrm{apex} \mathrm{for} \sim 8 \mathrm{hrs}$. Moreover, three observations that showed an inconsistent anti-correlated lag remained at the HB and upper NB together with a few other observations having a low CC (Table 1). We also found that out of the 23 detected lags, only 4 lags are negative soft lags which tend to appear near the hard apex. Figure 3 shows the histograms for the 128 segments of the 74 observations with respect to their locations on the Z-track. The observations showing smaller CCF than \pm 0.4 were included in the histograms of anti-correlation data. It is evident that most of the anti-correlated lags are localized along the HB and hard apex.

To investigate the possibility that two uncorrelated light curves give a correlation purely by chance, we have performed simulations by generating two long independent light curves $\left(2^{20} \mathrm{~s}\right)$ based on the method of Timmer and Koenig (1995). The observation of ObsID 30042-01-11-00 showed the highest anti-correlation coefficient $(\sim-0.9)$ and hence we used the parameters of the soft band light curve (i.e., mean count rate of 5229 counts s$^{-1}$, standard deviation of 118 counts $\mathrm{s}^{-1}$, and power-law index of PDS $\beta=1.76$ ) and hard band light curve (i.e. mean count rate of 191 counts $\mathrm{s}^{-1}$, standard deviation of 11 counts $\mathrm{s}^{-1}$, and power-law index of $\operatorname{PDS} \beta=2.85$ ) to obtain the two simulated light curves. The power-law index was obtained from the observation by fitting a power-law model to the corresponding PDS. Then the two simulated light curves were cross-correlated, where the simulated curves have a bin size of $32 \mathrm{~s}$ and a segment length of 3000 s. The CCF and delay histograms are shown in the Figure 4. The number of segments showing the correlation coefficient of $\leq-0.9$ are low (22 out of 348 segments). It was also found that in the simulations the number of segments showing a low level of correlation (or no correlation) is also very low (see Figure 4). 


\section{Spectral Analysis Results}

\subsection{Model-independent study of spectral variation}

There is an idea that the radiative process changes during the lag time. It is therefore important to confirm whether the spectrum varies during the anti-correlated lag times. To see such a change, we extracted energy spectra from the initial and final segments of the light curves and superposed them. Figure 5 shows the superposed spectra (left panels) along with their spectral ratios (right panels) for four observations that are located at different branch positions. The spectral ratios show a spectral pivoting around $\sim 3-10 \mathrm{keV}$ and its energy changes depending on the location on HID. Similar kind of spectral pivoting was found in BHXBs (Choudhury et al. 2005; Sriram et al. 2007; Sriram et al. 2009) and Cyg X-2 (Lei et al. 2008). We also found that for positively correlated observations the spectra do not show such a spectral pivoting, similar to the result by Lei et al. (2008). For example, we found no spectral pivoting in the spectra of ObsID 30042-01-12-00 in which the CCF ( 0.3) shows no correlation or a low level of correlation. This model independent analysis suggests that the underlying radiative process changes during the detected anti-correlated lag times. It is interesting to note that the pivoting energy is close to 10 $\mathrm{keV}$ at the $\mathrm{FB} / \mathrm{soft}$ apex and the spectral ratios show a sharp and discontinuous feature at $10-15$ $\mathrm{keV}$. It is tempting to speculate that fast spectral pivoting at this energy might be the reason for the unexplained spectral feature detected at $10 \mathrm{keV}$ at the FB (Jackson et al. 2009; Asai et al. 1994).

\subsection{Spectral variation in horizontal branch}

We carried out spectral analysis on the various positions of HB. For this purpose, we selected the data of ObsID 30042-01-13-00 which spans from the hard apex to upper HB. In the observation, the soft and hard X-rays were anti-correlated: the decrease in the soft X-rays accompanied by a simultaneous increase in the hard X-rays (top panel in Figure 6). A delay was 
also observed for each segment of the light curve together with a variation of the spectral pivoting energy (Table 3), except the segment B which does not show any correlation/anti-correlation (CCF $\leq 0.2$ ). We extracted energy spectra for the four segments marked as A, B, C, and D in the light curve of Figure 6 and did a model independent comparison for the spectra which strongly suggest a change in the radiative process (the second panel of Figure 6). We then fitted two component models to the spectral data of $3-25 \mathrm{keV}$, "diskBB +CompTT" (diskBB model: Mitsuda et al. 1984; CompTT model: Titarchuk 1994) and "BB+CompTT", which are often applied to fit the spectra of Z-type sources (Done et al. 2002; Agrawal \& Sreekumar 2003; Agrawal \& Misra 2009). Due to the absence of data below $3 \mathrm{keV}$, the absorption column density was fixed at $\mathrm{N}_{H}=$ $6.0 \times 10^{22} \mathrm{~cm}^{-2}$ (Jackson et al. 2009). The best-fit spectral parameters are summarized in Table 3 .

It is found that as the soft X-ray decreases from the segment A to D, both disk temperature $\left(\mathrm{kT}_{i n}=2.10 \mathrm{keV}\right.$ to $\left.\mathrm{kT}_{i n}=2.87 \mathrm{keV}\right)$ and black body temperature $\left(\mathrm{kT}_{b b}=1.51 \mathrm{keV}\right.$ to $\mathrm{kT}_{b b}=$ $2.04 \mathrm{keV})$ increases independently. The electron temperature $\left(\mathrm{kT}_{e}\right)$ does not vary significantly when the BB model is applied whereas its temperature considerably increases when the diskBB model is applied, i.e., as the source moves from the hard apex (segment A) to upper HB (segment $\mathrm{D})$, the electron temperature $\left(\mathrm{kT}_{e}\right)$ increases whereas the optical depth $(\tau)$ decreases (Table 3$)$. The unfolded spectra shown in the third panel from the top of Figure 6 were obtained with the diskBB+CompTT model. From this spectral analysis, it is inferred that both Comptonizing medium (or Compton cloud) and disk properties change when the source moves along the track (from hard apex to upper HB). In case that the BB+CompTT model is applied, the black body temperature only varies significantly (Table 3 ).

We analyzed PDSs for each of the segments to fully understand the physical changes, whether there is a dynamical change of the disk or not (i.e. radial movement of the inner disk front; Done et al. 2007). It was found that the four segments (A, B, C, and D) show HBOs having different frequencies (Table 3, the bottom panels of Figure 6), which are a general characteristic 
of $\mathrm{Z}$ sources. From the segment A to D, the HBO frequency changes from $v_{H B O}=51 \mathrm{~Hz}$ to $v_{H B O}$ $=21 \mathrm{~Hz}$. We found that as the source moves from the hard apex to upper HB the HBO frequency decreases, which is in agreement with the result of Jonker et al. (2002) who found that low frequency QPOs $(15-50 \mathrm{~Hz})$ increase from the upper HB to upper NB in GX 5-1. In general, the relatively high frequency QPOs are supposed to originate closer to NS/BH and low frequency QPOs originate away from NS/BH in the Keplerian disk. The dynamical variability analysis indicates that at the hard apex the inner disk front is close to NS $\left(v_{H B O}=51 \mathrm{~Hz}\right)$ and at the upper $\mathrm{HB}$ the disk is away from NS $\left(v_{H B O}=21 \mathrm{~Hz}\right)$.

In brief, as the source traverses from the hard apex (section A) to upper HB (section D), the observed delay decreases, HBO frequency decreases, pivoting energy increases, black body temperature and flux increase, and the Comptonizing region changes from a low temperature and high optical depth compact plasma to a high temperature and low optical depth plasma. If we assume that the Compton cloud resides inside a truncated disk and these changes are interpreted as an increase in the truncation radius, these findings indicate that there exists a small black body region possibly at the surface of the neutron star, which is unrelated to the size of the truncated disk (disk temperature should have considerably reduced, if it is related to the truncation radius). The delay time, then, should pertain to the adjusting timescale for the Compton cloud in the new disk configuration, which appears to be greater for the high optical depth plasma.

\subsection{Frequency variation of $\mathrm{HBOs}$}

In the case of BHXBs, it is known that during the lags the QPO frequencies shift to higher or lower frequencies (Choudhury et al. 2005; Sriram et al. 2007; Sriram et al. 2009; Sriram et al. 2010). The origin of HBOs and NBOs in NS-LMXBs are not well known, particularly the generating mechanism of low frequency QPOs is poorly understood. These QPOs can originate either from the Corona (Chakrabarti \& Manickam 2000; Titarchuk \& Fiorito 2004) or from the 
disk (Done et al. 2007; Ingram \& Done 2010). To see the high frequency variation of HBOs in detail, we select ObsID 20053-02-01-01 which shows a negative lag of $\sim-250$ s, because Wijnands et al. (1998) already reported the $\mathrm{kHz}$ QPO of $\sim 500 \mathrm{~Hz}$ for this observation.

We analyzed the PDS for ObsID 20053-02-01-01 shown in Figure 7 (which is obtained from the initial (A) and final (B) segment of the light curve in Figure 1) and found that there is a significant frequency variation in the HBOs from $v_{H B O}=23 \mathrm{~Hz}$ (segment A) to $v_{H B O}=18 \mathrm{~Hz}$ (segment B), suggesting a dynamical change in the accretion disk. Wijnands et al. (1998) found that there are $\mathrm{kHz}$ QPOs in the higher energy band $(8.6-60.0 \mathrm{keV})$ but not in the lower energies. For comparison, we extracted the Leahy power PDS for the segment A and B in high (8.6 $60.0 \mathrm{keV})$ and low $(6.9-8.3 \mathrm{keV})$ energies and plotted those in Figure 7. As shown in Figure 7 and Table 4, there exist $\mathrm{kHz}$ QPOs at $\sim 590 \mathrm{~Hz}$ (segment A) and $\sim 550 \mathrm{~Hz}$ (segment B) in the higher energy band only, confirming the earlier result by Wijnands et al. (1998). Apart from the frequency variation in HBOs, we found that the frequency in $\mathrm{kHz}$ QPOs tends to decrease from $\sim 590 \mathrm{~Hz}$ to $\sim 550 \mathrm{~Hz}$ although this tendency is marginally significant.

We also investigated a spectral variation between the two segments. For this, we applied the diskBB+CompTT model to the spectra and extracted the unfolded spectra shown in Figure 7. The best-fit parameters are listed in Table 4. By applying the best-fit parameters of the spectrum A to $\mathrm{B}$, we obtained the $\chi^{2}$-value of $\chi^{2} / \mathrm{dof}=3027 / 47$ which clearly indicates that the spectrum has changed. We found that the disk and thermal Comptoinzation parameters have changed significantly. For example, the disk temperature has increased from $\mathrm{kT}_{\text {in }}=2.84 \mathrm{keV}$ to $\mathrm{kT}_{\text {in }}=$ $2.95 \mathrm{keV}$ along with the increase of electron temperature from $\mathrm{kT}_{e}=7 \mathrm{keV}$ to $\mathrm{kT}_{e}=13 \mathrm{keV}$. Similar kind of variation in electron temperature was reported for GRS 1915+105 in which a lag was detected (Sriram et al. 2007). The changes in HBO (from $18 \mathrm{~Hz}$ to $23 \mathrm{~Hz}$ ) and kHz QPO (590 $\mathrm{Hz}-550 \mathrm{~Hz})$ along with the electron temperature $(7-13 \mathrm{keV})$ clearly indicates that the Compton cloud size is increasing which can be the cause of the observed soft X-ray lag in this observation. 


\section{Discussion and Conclusion}

\subsection{Anti-correlated lags and their positions on HID}

We investigated the CCFs of GX 5-1 between soft (2-5 keV) and hard (16-30 keV) X-ray light curves. Lei et al. (2008) detected anti-correlated hard and soft lags for Cyg X-2 and they suggested that the accretion disk in the source could be truncated. O' Brien et al. (2004) also found an anti-correlated variation for Cyg X-2 between optical and X-ray. Earlier studies of a few BHXBs reported a few $100-1000 \mathrm{~s}$ lags in the CCF of soft and hard X-ray bands and the detailed temporal and spectral analysis favored a truncated accretion disk geometry too (Choudhury et al. 2005; Sriram et al. 2007; Sriram et al. 2009; Sriram et al. 2010). The truncated accretion disk model is often used to explain the spectral and temporal features of the hard states observed in BHXBs (Done et al. 2007) but some observational results show that the disk may not be truncated (Miller et al. 2006; Rykoff et al. 2007). Done \& Gierlinski (2006) argued that non-truncated disk models can be ruled out based on the outflowing disk wind processes. The broad iron line in low/hard state was presented as the evidence of non-truncated disk (Miller et al. 2006) but a re-analysis of the data showed that the line is instead narrow and consistent with the truncated disk geometry (Done \& Diaz Trigo 2010). The physical condition is relatively more complex in neutron star LMXBs. We carried out such a similar study for GX 5-1 because the longer time lags seem to provide an opportunity to explore the geometry of accretion disk in LMXBs.

Our study on CCFs showed that on most occasions the soft and hard X-ray light curves are positively or loosely correlated but on a few occasions the CCFs show statistically significant anti-correlated hard (mostly) and soft X-ray lags (Figure 1, Table 1, and Table 2). Since the hard X-rays are Comptonized from the soft X-rays, they should be correlated each other but this and previous studies for a few BHXBs and Cyg X-2 indicate that a re-adjustment in the emission geometry and radiation process is necessary to interpret the anti-correlations. The hard X-ray lag means that the arrivals of hard X-rays are delayed to the observer compared with the soft X-rays. 
We found that the centroid frequency in HBOs changes toward the lower frequency during the lag (Figure 6). This result strongly suggests that the accretion disk dynamically changes during the lag. The frequency shift could be due either to an outward radial movement of disk (Done et al. 2007) or to change in the oscillation of Compton cloud (Chakrabarti \& Manickam 2000). The difference in lag timescale implies that the radial movement of disk occurs differently at various radii/locations which can effectively affect on the properties of Compton cloud. If we assume that the mass accretion rate is the primary parameter to determine the location on HID, then the detected lags should be related somehow to the viscous timescale during which the accretion disk is re-adjusted because the other timescales like dynamical and thermal timescales in accretion disk are less in magnitude (Frank et al. 2002). At the same time the observed lags are much smaller than the viscous timescale of the full accretion disk, which is generally of the order of a few tens to hundred days (often assumed to be the duration of an outburst) (Sturner \& Shrader 2005 ), and hence we speculate that the inner disk front movement occurs at a much smaller radius. However, based on recent results (Lin et al. 2009; Homan et al. 2010), mass accretion rate was ruled out as the primary driving physical parameter to cause a $\mathrm{Z}$ shape in HID hence we suggest that the observed lags could be due to change in the size of the Comptonizing region. Based on the study of black hole source XTE J1550-564, Homan et al. (2001) found that independent of mass accretion rate, the size of the Comptoinzing region could be potentially possible physical factor to determine the state of the source.

Our observed lags may not be explained by invoking just a simple radiative process such as Comptonization. There are several models which can account for small ( $\leq 1 \mathrm{~s})$ timescale lags (Kazanas et al. 1997; Nowak et al. 1999; Bottcher \& Liang 1999). In these models, some of the soft X-rays radiating from the NS surface, accretion disk, or a soft X-ray source in the form of a dense blob made of disk material get Comptonized by hot electrons $\left(\sim 10^{8} \mathrm{~K}\right)$ and are converted into the hard X-rays. During this process the hard X-rays can be delayed by $\leq 1 \mathrm{~s}$ over the soft X-rays that do not suffer from the Comptonization. However, we detected about hundred times 
longer lags from GX 5-1. These lags can be explained if we assume that the accretion disk is truncated. Jackson et al. (2009) carried out a detailed spectral analysis for GX 5-1 and they suggested that the existence of a truncated accretion disk structure cannot be ruled out at the upper NB and HB.

Similar to Cyg X-2, we found that most of the anti-correlated lag observations are located at the HB and upper NB (Table 2, Figure 2 and Figure 3). As such there is no exact relation between observed delays and corresponding positions on HID but we suggest that especially at the HB and upper NB, the disk forms a thin structure and corona forms an inner quasi-spherical shape making a truncated accretion disk geometry. Fender (1999) found that for black hole binaries the physical body forming the Comptonizing corona is the base of the jet. The radio observations show that a jet component is possibly present close to the central region of the disk when the source is at the hard apex and HB (Penninx 1989; Berendsen et al. 2000). Hence we suggest that the detected lags indirectly indicate a truncated accretion disk in GX 5-1 when the source is at the HB and NB. Since the mass of NS in GX 5-1 is not known, it is difficult to estimate the truncation radii. If we assume the properties of Cyg X-2, the truncation radius would be slightly less than 18 NS radius (Lei et al. 2008). It is because, GX 5-1 is fainter than Cyg X-2 and the viscous timescale is proportional to the inverse square of mass accretion rate $\left(\mathrm{t}_{v i s} \propto \dot{M}^{-2}\right)$.

\subsection{Spectral variations}

The model independent spectral analysis in $\$ 4.1$ suggests that the radiative process changes during the lag time which can be clearly seen in the spectral ratios in Figure 5. The spectral ratios show that the intensity variation accompanies a spectral hardening in three observations but accompanies a spectral softening in the other observation. This spectral pivoting is a strong signature of an opposite radiative process change in the soft and hard X-ray emitting regions. Such a spectral pivoting was not observed when the source shows a correlated intensity variation. 
Lei et al. (2008) reported a similar result too.

We analyzed a spectral and PDS evolution from the hard apex to upper HB (segment A to D) and obtained that as the HBO frequency decreases $(v=51 \mathrm{~Hz}$ at the hard apex to $v=21 \mathrm{~Hz}$ at the upper $\mathrm{HB}$ ), the coronal temperature increases along with a decrease in the optical depth (Table 2). Simultaneously, the increase in the disk temperature is accompanied by a decrease in the disk normalization which is a close measure of the inner disk radius. In addition, the variation in the HBO frequency is consistent with the variation of coronal parameters (Chakrabarti \& Manickam 2000; Titarchuk and Shaposhnikov 2005) but is inconsistent with the variation of disk or black body parameters. This discrepancy also arises when we relate the HBO frequency variation with that of diskBB/BB spectral parameters, i.e., in the frame of a truncated accretion geometry, as the disk moves inward (in a dynamical point of view, $v_{H B O}=21 \mathrm{~Hz}$ to $51 \mathrm{~Hz}$ ), the disk temperature is supposed to increase but it is decreasing. It was found that in a strong Comptonization the obtained disk temperature should be lowered (Done \& Kubota 2004).

Conventionally as the disk approaches close to the NS, the QPO frequency increases, disk normalization decreases and the increase in soft X-rays cools the relatively hot corona. Since the disk is in a deeper gravitational potential, the inner disk temperature should be relatively high when compared to the disk temperature at lower QPO frequency. But our analysis shows that the disk temperature is low along with a high disk normalization value at higher HBO frequency $(\sim 51 \mathrm{~Hz})$ whereas at low HBO frequency $(\sim 21 \mathrm{~Hz})$ the disk temperature is high with a low normalization value. Even the black body temperature is found to be low at $51 \mathrm{~Hz}$ compared with the BB temperature at $21 \mathrm{~Hz}$. This decrease in the soft component temperature is similar to the result by Jackson et al. (2009) and Balucinska-Church et al. (2010) who found that, for GX 5-1 and other Z-track sources, the black body temperature (BB) decreases as the respective source moves from the upper HB to hard apex. We suggest that the low inner disk temperature and high disk normalization at $51 \mathrm{~Hz} \mathrm{HBO}$ (which is implying the disk is truncated at large radii) 
may be due to an instability caused by the radiation pressure ( $\left.\propto \mathrm{T}^{4}\right)$ (Jackson et al. 2009). But in this scenario, if the inner region of the disk outflows, the $51 \mathrm{~Hz} \mathrm{HBO}$ should not be observed. The other possible scenario to account for the $51 \mathrm{~Hz} \mathrm{HBO}$ could be that some portion of the coronal material condenses back to form the disk in the inner region and which is not in thermal equilibrium. Such type of re-condensation of corona in the intermediate/SPL state is explored in black hole binaries (Meyer et al. 2007) and the observational signatures were found in GX 339-4 (Sriram et al. 2010). However, such re-condensation models are yet to be explored for neutron star sources. Alternatively, the observed black body component may not be arising from the inner parts of the truncated disk; rather they may be a part of the NS surface.

We also carried out the spectral and PDS analysis for ObsID 20053-02-01-01 in which a soft X-ray lag $(\sim-250 \mathrm{~s})$ was detected. It was found that the HBO frequency shifts from $v=23$ $\mathrm{Hz}$ (segment A) to $v=18 \mathrm{~Hz}$ (segment $\mathrm{B})$ whereas the disk temperature increases from $\mathrm{kT}_{\text {in }}=$ $2.84 \mathrm{keV}$ to $\mathrm{kT}_{\text {in }}=2.95 \mathrm{keV}$ with a simultaneous decrease in the disk normalization (Table 4). During these change, the electron temperature increases from $\mathrm{kT}_{e}=7 \mathrm{keV}$ to $\mathrm{kT}_{e}=13 \mathrm{keV}$ with a decrease in the optical depth. The frequency shift suggests that the disk is moving away from the NS along with an increase in the electron temperature, which is generally observed in BHXBs. But the disk temperature increases instead of decreasing. Again these changes can be explained in the frame work of a re-condensation process. The radial outward movement of disk (based on the HBO shift) and the expansion of Comptoinizing region could cause the soft X-ray lag. Li et al. (2007) explored such kind of disk movement theoretically and found that fluctuations in the inner region of the disk can disturb the given configuration and therefore this disturbance can cause the disk movement with a timescale of a few hundred seconds. 


\subsection{Truncated disk geometry: Overall analogies of lagged observations in BHXBs and Z sources}

In case of BHXBs, a detailed spectral analysis presented that the following parameters, e.g., the physical size of accretion disk, the electron temperature $\left(\mathrm{kT}_{e}\right)$, and the optical depth of Compton cloud, are all changing for the observations that lags are detected. It was suggested that as the inner front of accretion disk moves toward the black hole, the soft flux increases and cools the hot electrons which results in the relative decrease of hard X-ray flux (Sriram et al. 2007; Sriram et al. 2009; Sriram et al. 2010). The lag time is comparable to the viscous timescale during which the various physical and radiative process change. A recent study reported that the variation in disk emission leads to the hard X-ray emission of a time delay of $\leq 1 \mathrm{~s}$ or a few seconds, which may be caused by the fluctuations in the disk (Uttley et al. 2011). In our present work, both disk and Compton cloud properties are changing during the lag time of a few tens of seconds. In BHXBs, however, the soft X-rays originate from the disk whereas in NS systems the emitting site could be either NS surface or accretion disk.

The anti-correlated hard X-ray lags in BHXBs were detected when they are in the steep power-law (SPL) or intermediate states (for a review of the spectral states of BHXBs see e.g., Remillard \& McClintock 2006; Done et al. 2007; Belloni 2010). The intermediate frequency QPOs $(\geq 1 \mathrm{~Hz})$ and $\mathrm{kHz}$ QPOs in BHXBs were detected only in these states and radio studies

showed that the jet component switches on/off during these states (Fender et al. 2009). A detailed radio study provided a substantial observational evidence for the similarity between Z-type sources and transient black holes at very high accretion rates (Migliari \& Fender 2006). Moreover, the Z-type source luminosities close to the Eddington limit $\left(\mathrm{L} \sim \mathrm{L}_{E d d}\right)$ which is often observed in the SPL state of BHXBs (Done et al. 2007). The temporal and spectral properties in the SPL/IM state together with the properties in radio emissions are analogous to those for Z-type sources in the HB and upper NB. One of the important spectral properties in the SPL state is the presence of 
compact corona (Done \& Kubota 2006; Sriram et al. 2007). Such signatures for Z-type sources were also revealed by the Integral observations (Paizis et al. 2005, 2006). These analogies in Z-type sources and BHXBs suggest that the truncated accretion disk is the most probable physical configuration which can consistently explain the various spectro-temporal properties.

In conclusion, we detected anti-correlated hard and soft time lags of the order of a few tens to hundred seconds in the Z-type source GX 5-1. We found that most of the lagged observations are located at the HB and upper NB. The magnitude of the lag corresponds to the viscous timescale of the inner accretion disk and hence indicates the viscous re-adjustment timescale of a hypothetical truncation radius. The change in QPO frequency and spectral parameters also support this conclusion. On the different branches, the spectral and temporal behaviors suggest that during the observations the accretion disk is dynamically changing, most probably due to the different mass accretion rate. However we do not find any tight correlation between delay times and branch positions on HID. The PDS analysis shows that the shifts in HBO frequencies are strongly correlated with the thermal Comptonization parameters instead of the disk parameters, which suggests that the HBOs are occurring at the hard X-ray emission region. The spectral and HBO frequency changes favor the scenario where the inner disk is truncated due to the radiation pressure at the HB and hard apex. The observed lag and spectral changes made it substantially evident that the geometry of the accretion disk is truncated. Overall, from the upper HB to hard apex, the spectral and PDS evolution suggest that there is a slightly different physical/geometrical condition compared to BHXBs. In BHXBs, as the QPO frequency decreases, the disk temperature decreases but the coronal temperature increases. On the other hand, in GX 5-1, both the disk and coronal temperatures increase as the QPO frequency decreases. This difference is most probably due to the existence of a hard surface in neutron stars which can provide a plausible site for the thermal emission.

We thank the anonymous referee for the very useful comments. This research has made use 
of data obtained through the HEASARC Online Service, provided by the NASA/GSFC, in support of NASA High Energy Astrophysics Programs. 

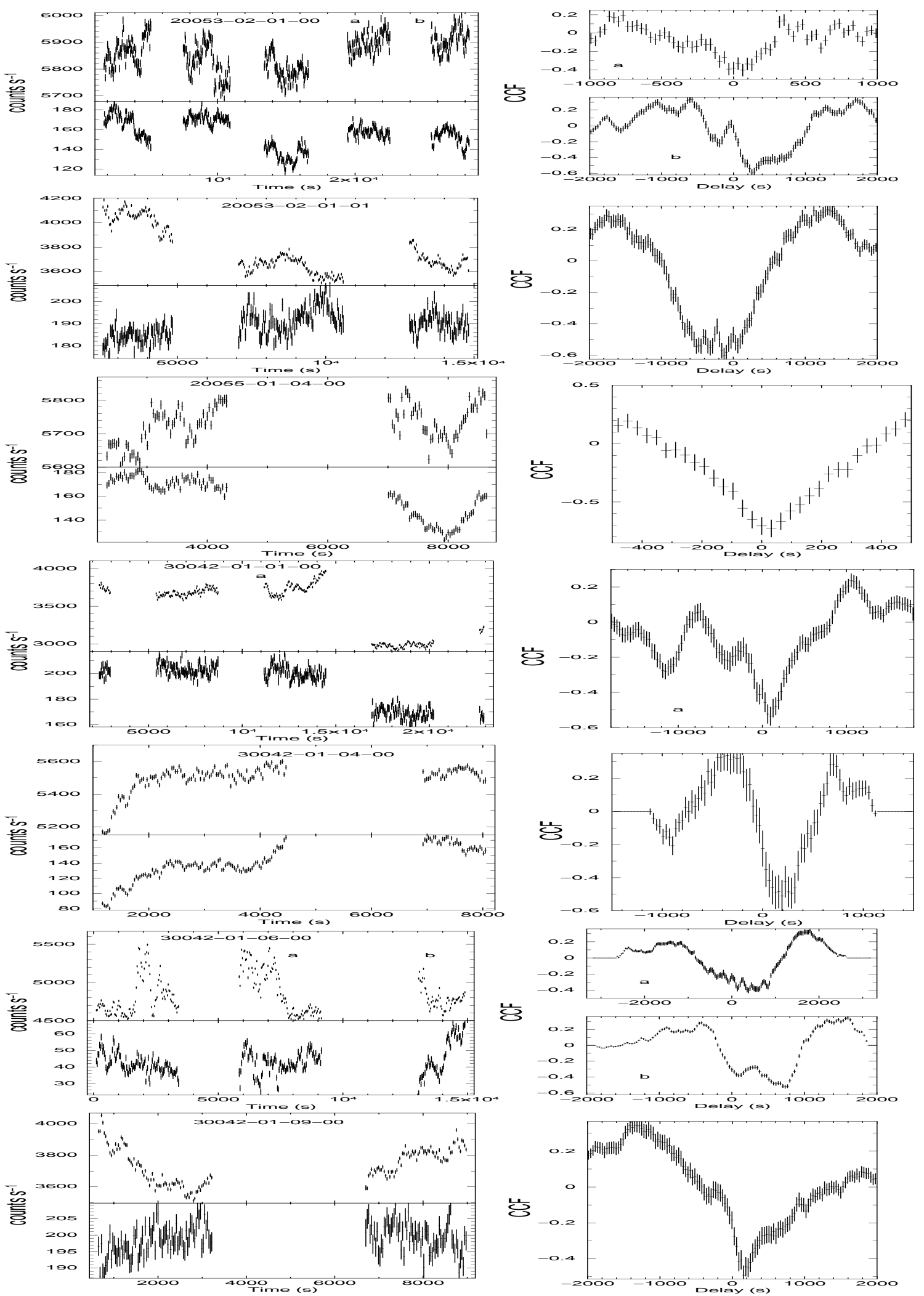

Fig. 1. - The background subtracted light curves of 17 observations for which anti-correlated X-ray lags are detected (left panels; top: $2-5$ $\mathrm{keV}$ and bottom: $16-30 \mathrm{keV}$ with bin size of $32 \mathrm{~s}$ ) and the corresponding cross-correlation functions (right panels). The ObsId is labeled for each observation. Symbols ' $a$ ' and 'b' correspond to the individual segments for which delays are detected. 

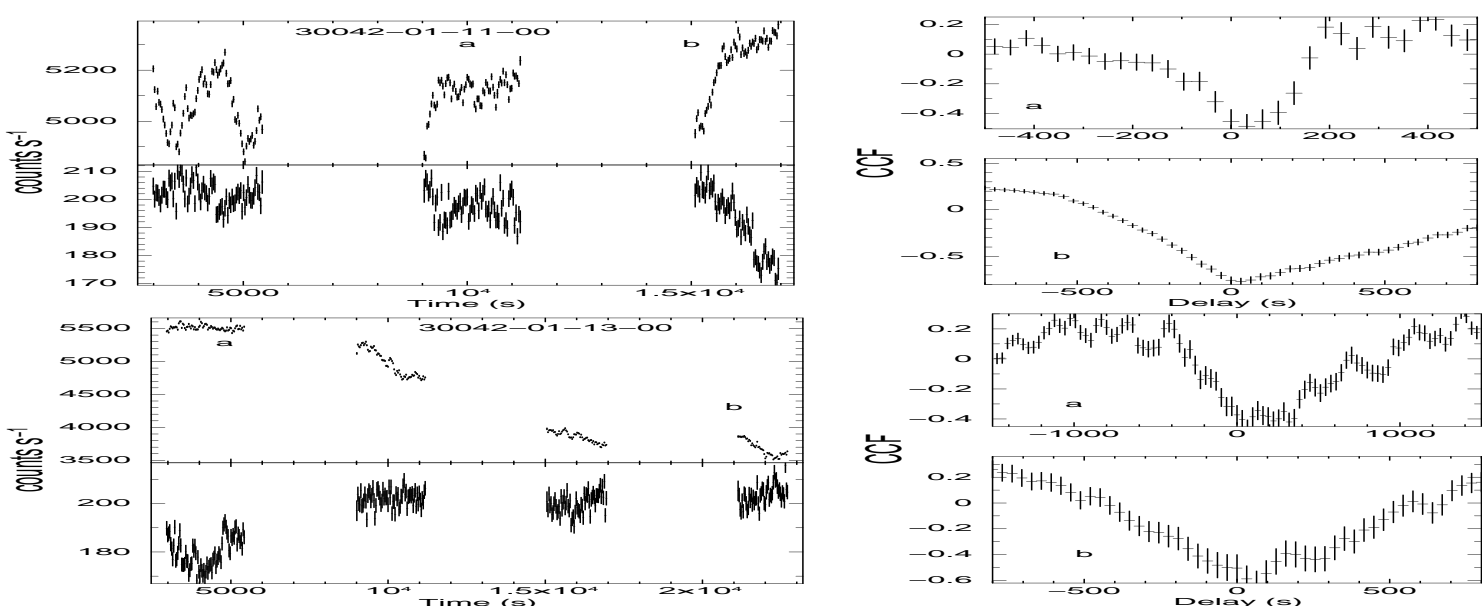

宅
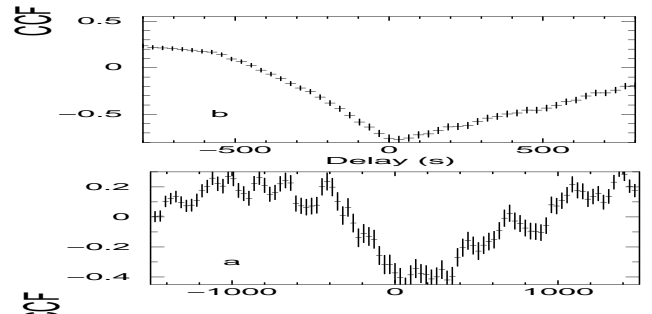

롱
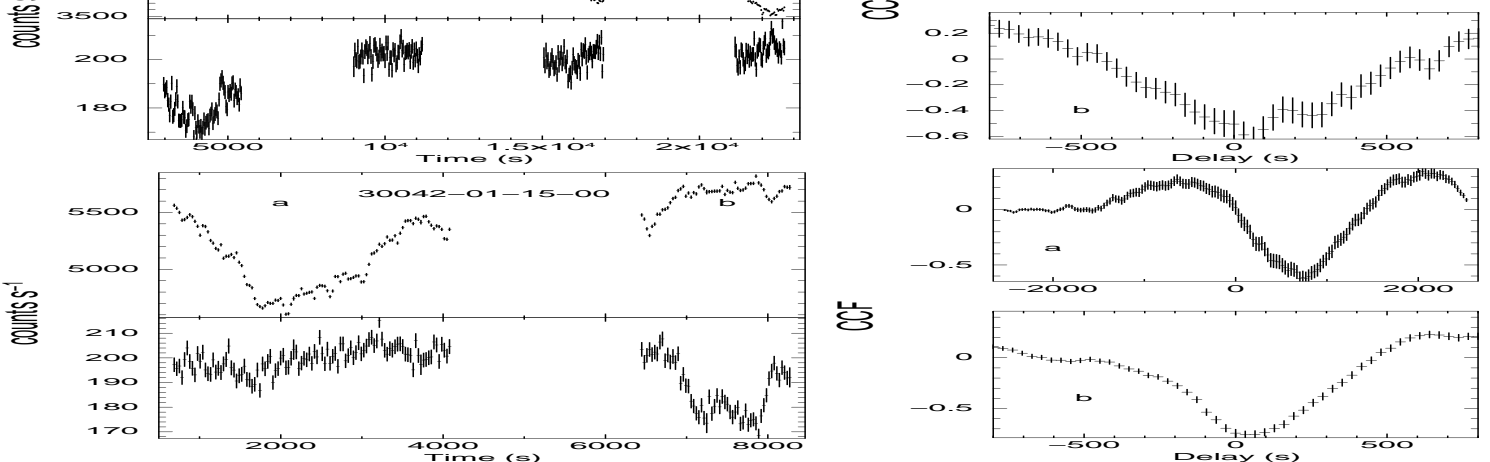

岁
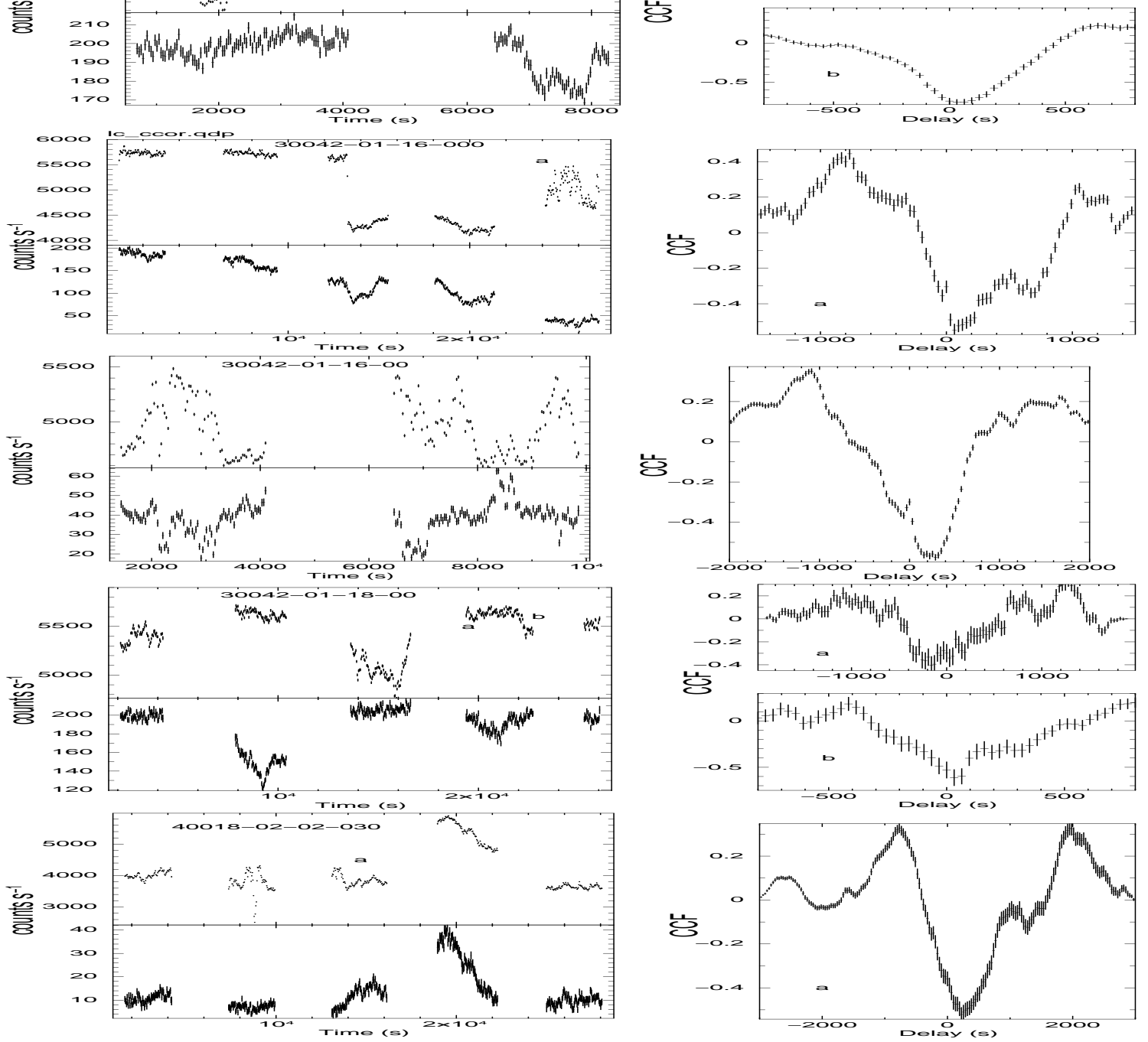

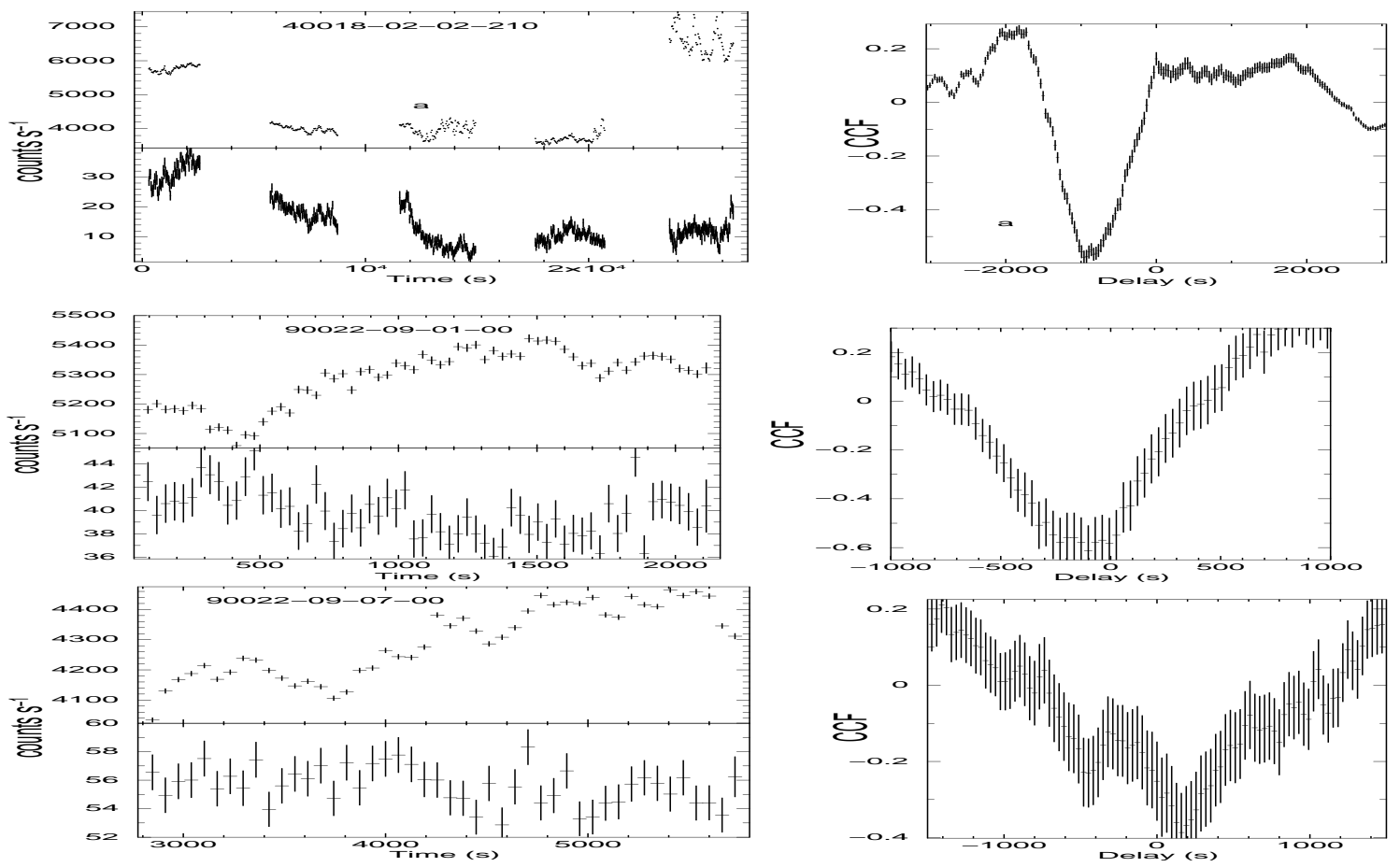


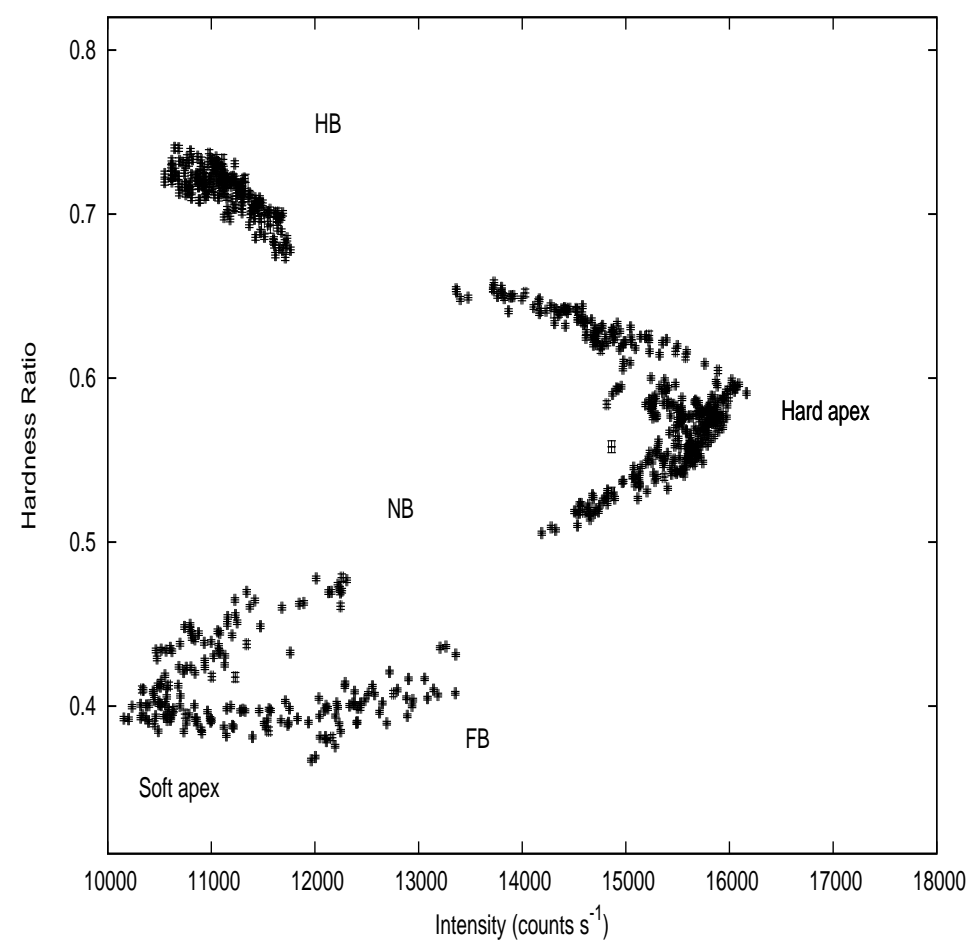

Fig. 2.- The Z-track is obtained using the observations of the period 1997-1998 for which anticorrelated lags are detected (see Table 2 for the position of each observation). 


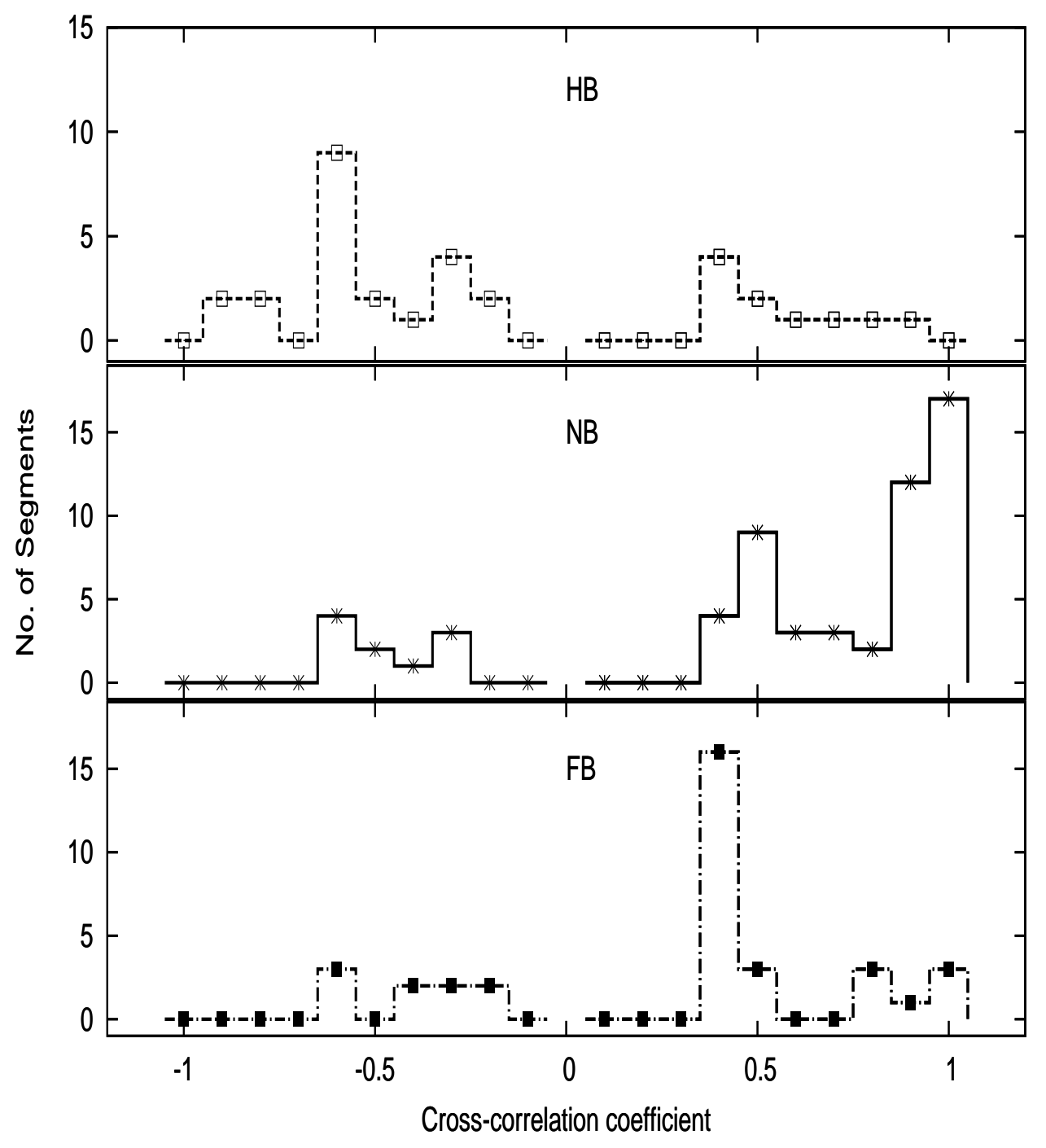

Fig. 3.- The histograms of all the observations based on their corresponding locations on the Z-track. Hard apex observations were included in HB and soft apex observations were included in FB to obtain the histogram. It is clear that most of the anti-correlated observations belongs to HB. 

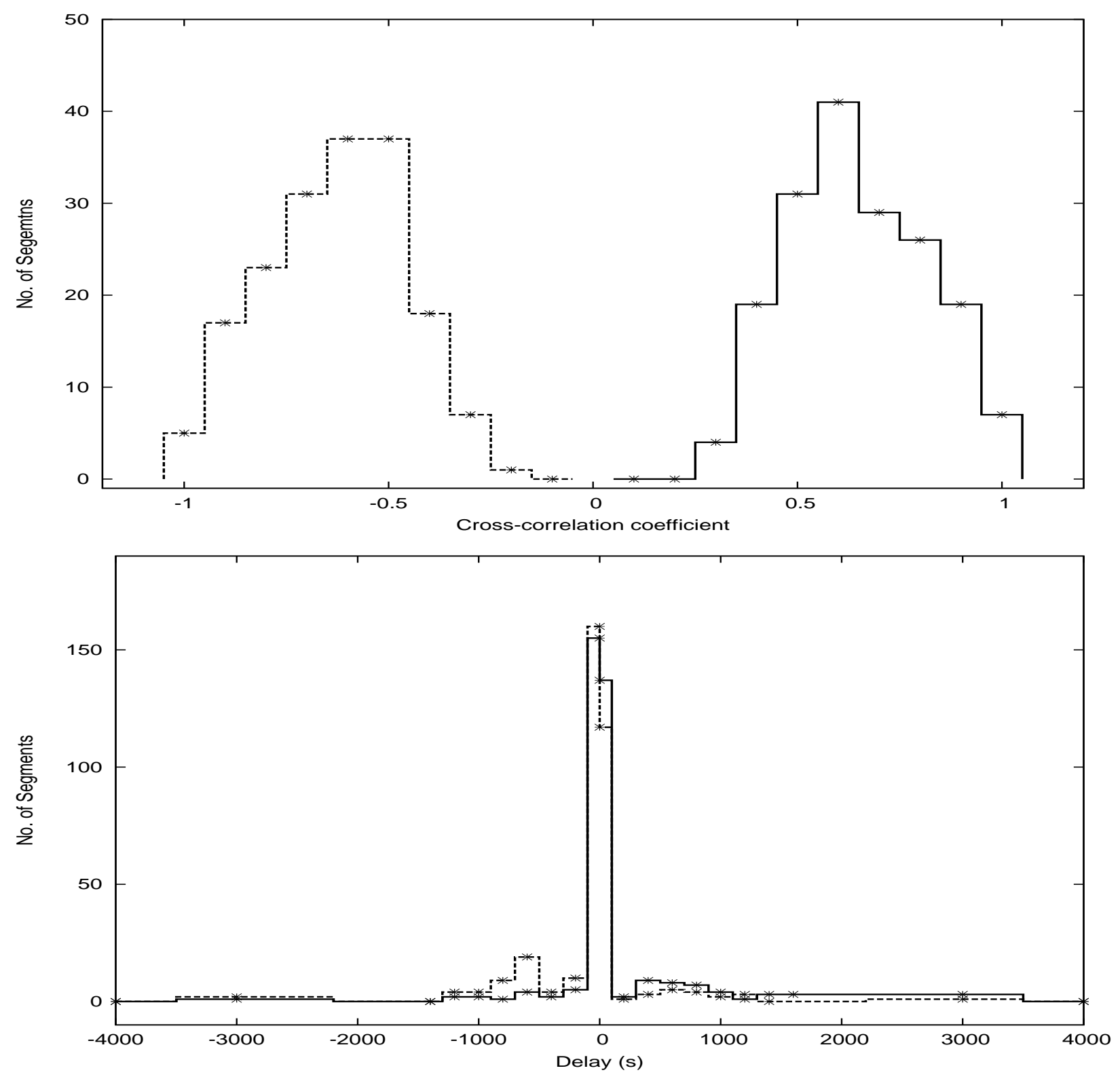

Fig. 4. - The upper panel shows the histogram of the CCF obtained from simulated light curves. The thick line shows the cross-correlation coefficient when the cross-correlation function is correlated and dashed line shows the same when the cross-correlation function is anti-correlated. The lower panel shows the histogram of the delay, where the thick line shows the delay when the cross-correlation function is correlated and dashed line shows the delay when the cross-correlation is anti-correlated (see text). 

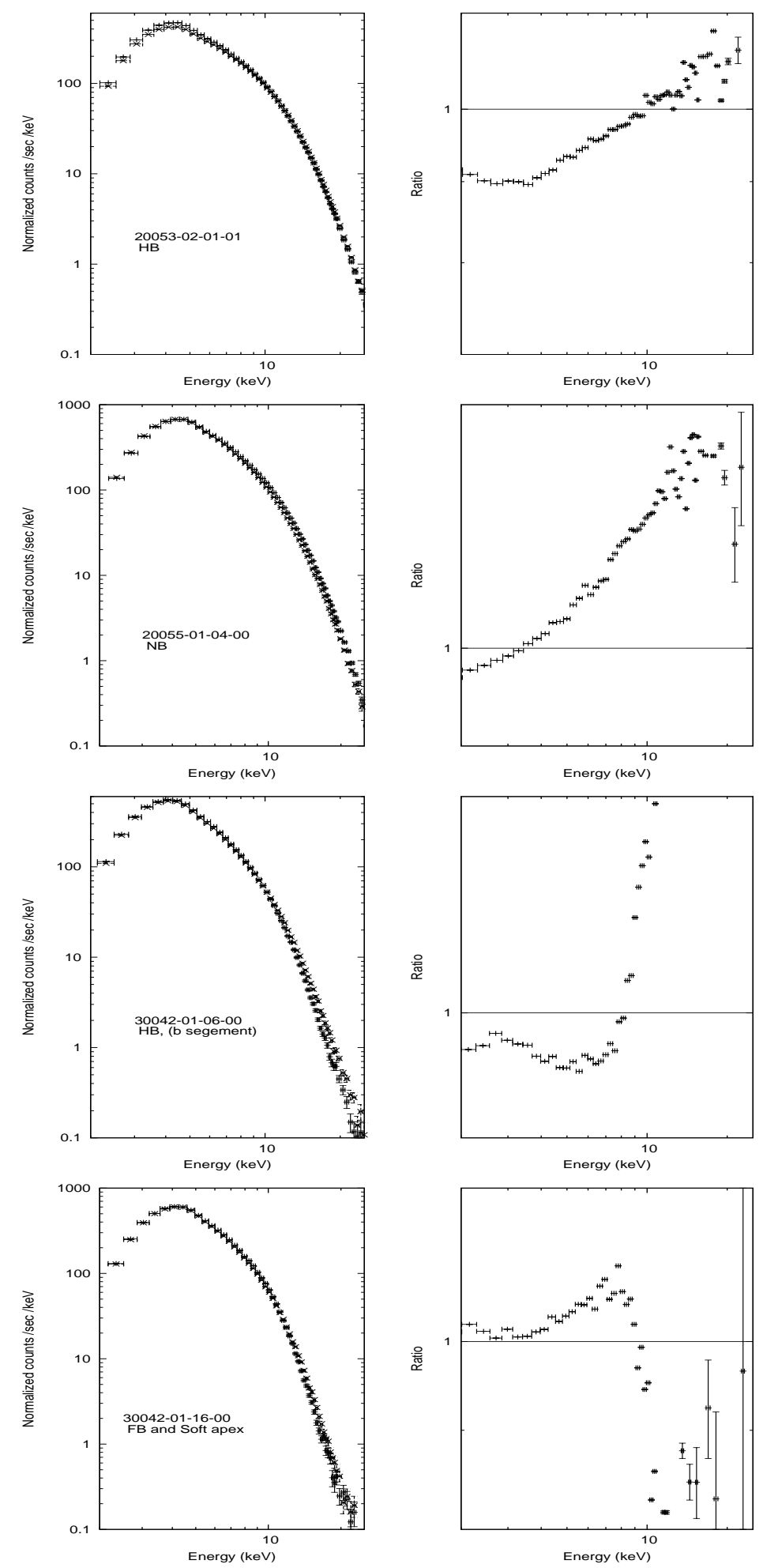

Fig. 5.- The energy spectra (left panels) along with their ratios (right panels) are shown for the observations that belong to different locations on the Z-track. The ObsID and the position on the Z-track are given in each figure of the spectra. 

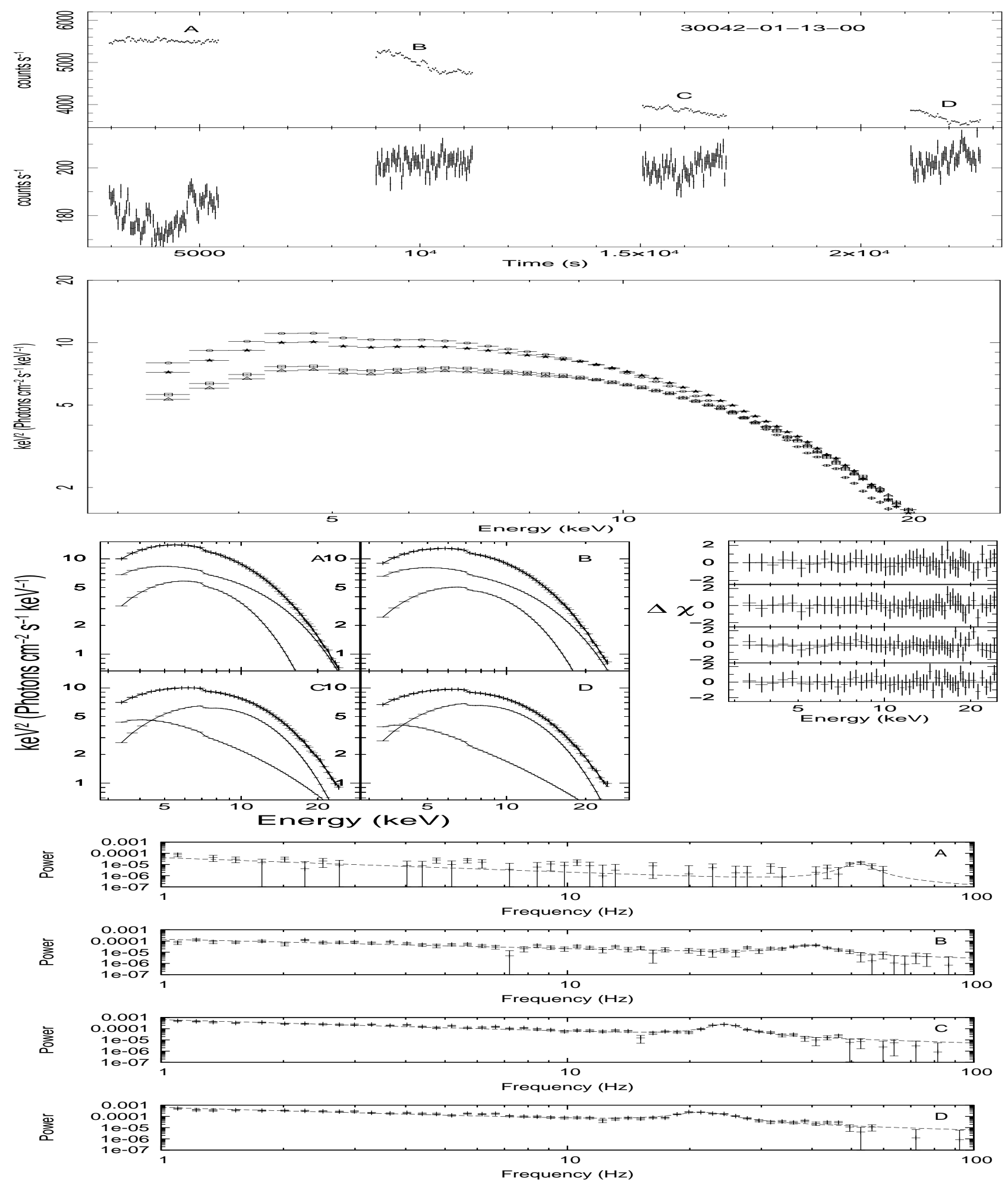

Fig. 6. - Top: The light curves of soft and hard X-rays for ObsID 30042-01-13-00. Second: Model independent spectral variation for the segments (A, B, C, and D) is shown. Third: The unfolded spectrum for each segment is plotted along with its model components (diskBB+CompTT) and the right panels show the $\Delta \chi$ of the corresponding best fit. Bottom: Power density spectrum $(2-5 \mathrm{keV})$ for each segment is shown, where the power is the normalized power in units of $(\mathrm{rms} / \mathrm{mean})^{2} / \mathrm{Hz}$. The dashed lines represent the best-fit model, power-law + Lorentzian. 

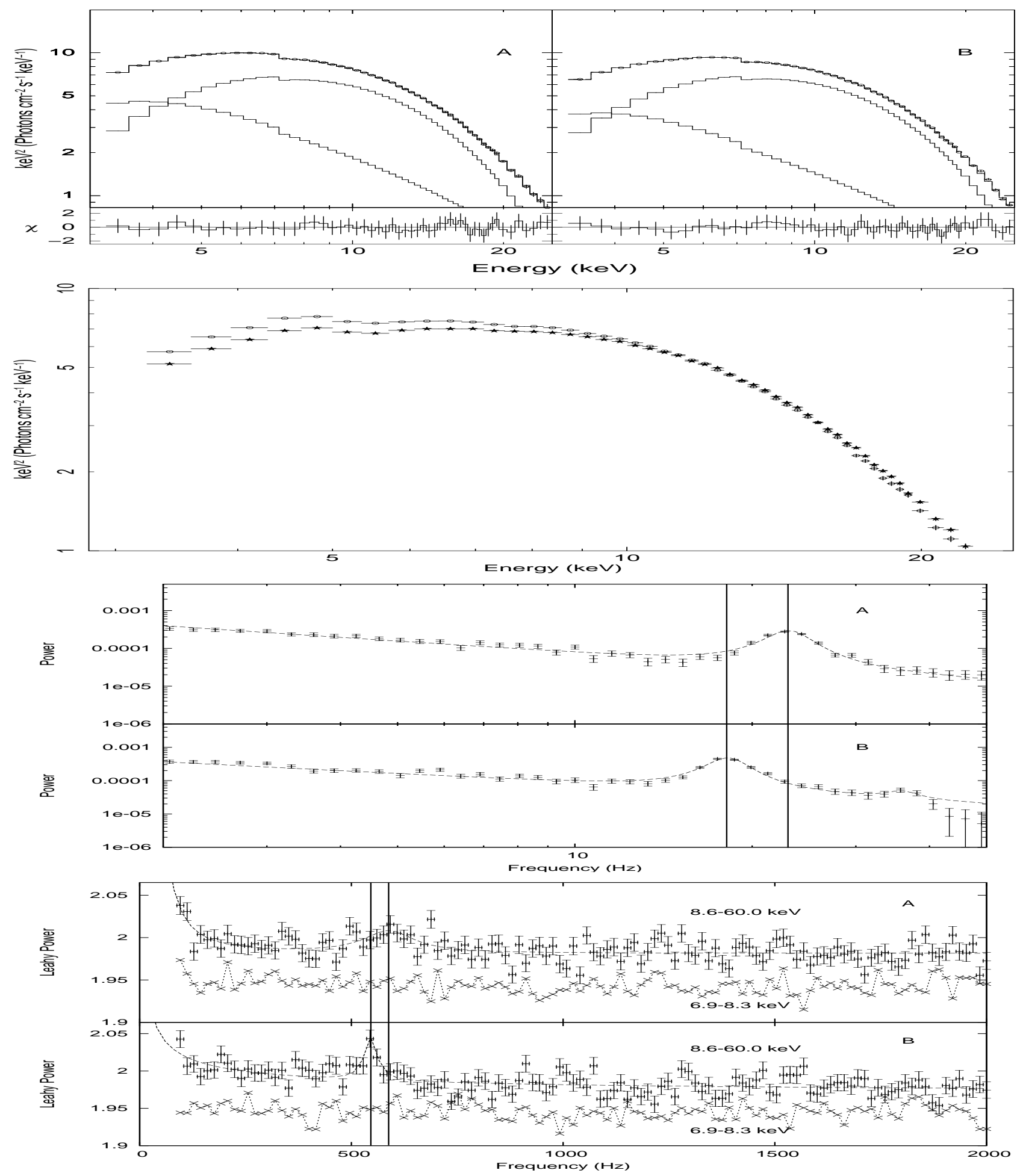

Fig. 7. - Top: Unfolded spectrum of the segment A and B for the ObsID 20053-02-01-01. Second panel shows the model independent change between A and B spectra. Third panel shows the PDS for the same segments, where the power is in units of ( $\mathrm{rms} / \mathrm{mean})^{2} / \mathrm{Hz}$ and the dashed lines represent the best-fit model, power-law + Lorentzian. The vertical solid lines represent the centroid frequencies of the individual segments. Bottom: Leahy power PDSs for the segment A and B in the energy band of $8.6-60 \mathrm{keV}$ and $6.9-8.3 \mathrm{keV}$. It is clear that the $\mathrm{kHz}$ QPOs are not present in the lower energy band. 
Table 1: Log of observations showing a positive correlation, a low level of correlation or an anti-correlation without any time lags. Hardness ratios and their branch positions on the Z-track are also listed. For long observations, the range of correlation coefficient (CC) are mentioned if the variations of CC are observed. The errors in CC (standard deviation) are ranging from $\pm 0.01- \pm 0.05$.

\begin{tabular}{|c|c|c|c|c|c|c|}
\hline No. & ObsID & Start Time & Stop Time & $\mathrm{CC}$ & Hardness Ratio & Branch Position on HID Plane \\
\hline 1 & $10061-02-01-00$ & 02-11-1996 08:34 & $16: 21$ & $0.65-0.96$ & $0.46-0.54$ & Lower and Upper NB \\
\hline 2 & $10063-02-01-00$ & 03-11-1996 20:30 & $02: 11$ & $0.83-0.95$ & $0.42-0.45$ & Lower NB \\
\hline 3 & 10061-02-02-000 & 06-11-1996 21:19 & $05: 13$ & $0.43-0.94$ & $0.38-0.54$ & Soft and Upper NB \\
\hline 4 & $10061-02-02-00$ & 07-11-1996 05:13 & $05: 34$ & 0.85 & $0.46-0.47$ & Lower and Middle NB \\
\hline 5 & $10061-02-03-00$ & 16-11-1996 00:55 & $08: 31$ & 0.85 & $0.60-0.67$ & Lower and Upper HB \\
\hline 6 & 20055-01-01-00 & 15-02-1997 08:32 & $10: 27$ & -0.73 & $0.59-0.63$ & Hard apex and Upper NB \\
\hline 7 & 20055-01-02-00 & 12-04-1997 19:00 & $20: 55$ & $0.25-0.4$ & $0.62-0.66$ & Lower and Middle HB \\
\hline 8 & 20055-01-03-00 & 29-05-1997 19:18 & $21: 55$ & $0.83--0.43$ & $0.38-0.44$ & Soft and Lower NB \\
\hline 9 & 20053-02-01-04 & 06-06-1997 00:28 & $02: 28$ & $0.42-0.73$ & $0.48-0.56$ & Upper NB \\
\hline 10 & 20053-02-02-00 & 25-07-1997 05:18 & 10:00 & 0.92 & $0.40-0.48$ & Lower and Middle NB \\
\hline 11 & 20053-02-01-02 & 25-07-1997 11:43 & $17: 29$ & $0.25-0.65$ & $0.45-0.65$ & Lower NB and Middle HB \\
\hline 12 & 20053-02-01-03 & 25-07-1997 18:23 & $20: 40$ & $0.35-0.8$ & $0.63-0.67$ & Lower and Middle HB \\
\hline 13 & 20055-01-05-00 & 21-09-1997 12:29 & $14: 24$ & 0.32 & $0.56-0.59$ & Upper NB and Hard apex \\
\hline 14 & $30042-01-02-00$ & 14-09-1998 00:25 & 03:02 & 0.30 & $0.40-0.55$ & Lower and Middle NB \\
\hline 15 & 30042-01-03-00 & 25-09-1998 03:28 & $08: 23$ & -0.52 & $0.60-0.66$ & Lower and Middle HB \\
\hline 16 & 30042-01-02-01 & 09-10-1998 03:20 & $06: 38$ & 0.98 & $0.40-0.53$ & Soft apex and Upper NB \\
\hline 17 & $30042-01-05-00$ & 14-10-1998 00:16 & $05: 59$ & $0.44-0.61$ & $0.38-0.42$ & Soft apex and Lower NB \\
\hline 18 & $30042-01-07-00$ & $31-10-199805: 23$ & $08: 45$ & 0.92 & $0.40-0.45$ & Soft apex and Lower NB \\
\hline 19 & 30042-01-08-01 & 02-11-1998 06:34 & $08-27$ & -0.85 & $0.74-0.78$ & Upper HB \\
\hline 20 & $30042-01-08-00$ & 03-11-1998 03:18 & 09:04 & $0.47-0.64$ & $0.62-0.66$ & Middle HB \\
\hline 21 & $30042-01-10-00$ & 08-11-1998 06:36 & $07: 25$ & -0.30 & $0.69-0.72$ & Upper HB \\
\hline 22 & $30042-01-12-00$ & 10-11-1998 00:07 & $04: 28$ & $0.14-0.35$ & $0.66-0.72$ & Upper HB \\
\hline 23 & 30042-01-14-00 & 11-11-1998 03:16 & $07: 56$ & $0.27-0.44$ & $0.65-0.71$ & Upper HB \\
\hline 24 & $30042-01-17-00$ & 21-11-1998 11:47 & $18: 28$ & $0.67-0.92$ & $0.52-0.58$ & Middle and Upper NB \\
\hline 25 & $30042-01-19-00$ & 22-11-1998 06:35 & 09:06 & $0.42-0.58$ & $0.52-0.59$ & Upper NB \\
\hline 26 & $30042-01-20-00$ & 22-11-1998 09:47 & 11:17 & 0.73 & $0.51-0.54$ & Upper NB \\
\hline 27 & 40018-02-01-000 & 01-03-2000 15:30 & $23: 30$ & $0.33-0.72$ & $0.35-0.55$ & FB and Upper NB \\
\hline 28 & 40018-02-01-00 & 01-03-2000 23:30 & 03:43 & $0.42-0.65$ & $0.36-0.40$ & FB and Soft apex \\
\hline 29 & 40018-02-01-10 & 02-03-2000 04:30 & 05:08 & 0.94 & $0.38-0.45$ & FB and Lower NB \\
\hline 30 & 40018-02-01-03 & 02-03-2000 12:15 & $12: 59$ & 0.42 & $0.38-0.44$ & FB and Lower NB \\
\hline 31 & 40018-02-01-01 & 02-03-2000 15:29 & $16: 41$ & 0.68 & $0.36-0.41$ & FB and Lower NB \\
\hline 32 & 40018-02-01-04 & 02-03-2000 17:11 & 00:01 & $0.33-0.92$ & $0.38-0.51$ & FB and Middle NB \\
\hline 33 & 40018-02-02-10 & 03-03-2000 00:01 & 03:37 & -0.63 & $0.33-0.38$ & FB and Soft apex \\
\hline 34 & 40018-02-02-02 & 03-03-2000 07:25 & 13:04 & $0.76-0.9$ & $0.37-0.54$ & FB and Upper NB \\
\hline 35 & 40018-02-02-03 & 03-03-2000 23:24 & 03:39 & $0.87-0.18$ & $0.38-0.46$ & FB and Lower NB \\
\hline 36 & 40018-02-02-04 & 04-03-2000 05:57 & 13:00 & $0.42-0.95$ & $0.38-0.52$ & FB and Middle NB \\
\hline 37 & 40018-02-02-05 & 04-03-2000 13:52 & $14: 44$ & 0.84 & $0.36-0.44$ & FB and Lower NB \\
\hline 38 & 40018-02-02-21 & 05-03-2000 00:58 & 03:36 & $0.22-0.43$ & $0.38-0.42$ & FB and Lower NB \\
\hline 39 & 40018-02-02-14 & 05-03-2000 04:15 & 05:01 & 0.42 & $0.34-0.37$ & FB and Soft apex \\
\hline 40 & 40018-02-02-13 & 05-03-2000 17:56 & $23: 56$ & $0.24-0.91$ & $0.35-0.55$ & FB and Upper NB \\
\hline 41 & 40018-02-02-17 & 06-03-2000 02:34 & 03:33 & 0.25 & $0.38-0.43$ & FB and Lower NB \\
\hline 42 & $40018-02-02-22$ & 06-03-2000 04:10 & 05:09 & -0.43 & $0.40-0.42$ & Lower NB \\
\hline 43 & 40018-02-02-15 & $06-03-2000$ 05:46 & $06: 44$ & 0.91 & $0.40-0.46$ & Lower NB \\
\hline 44 & $40018-02-02-23$ & 06-03-2000 09:08 & $09: 56$ & 0.85 & $0.42-0.55$ & Lower and Upper NB \\
\hline 45 & 40018-02-02-16 & 06-03-2000 13:41 & $14: 40$ & 0.81 & $0.38-0.42$ & FB and Lower NB \\
\hline 46 & $40018-02-01-08$ & 06-03-2000 15:17 & $16: 29$ & 0.92 & $0.49-0.53$ & Lower and Upper NB \\
\hline 47 & 40018-02-02-09 & 06-03-2000 16:53 & 23:52 & $0.55-0.85$ & $0.40-0.53$ & Lower and Upper NB \\
\hline 48 & 40018-02-02-19 & 07-03-2000 05:39 & $09: 53$ & $0.41-0.92$ & $0.38-0.50$ & FB and Middle NB \\
\hline 49 & 50017-02-01-00 & 18-07-2000 07:29 & $10: 21$ & 0.92 & $0.47-0.53$ & Lower NB and Upper NB \\
\hline 50 & 50017-02-01-01 & 19-07-2000 10:57 & $12: 11$ & 0.54 & $0.38-0.40$ & FB and Lower NB \\
\hline 51 & 80105-09-01-00 & 24-09-2003 15:53 & $16: 54$ & 0.25 & $0.41-0.43$ & Lower NB \\
\hline 52 & 80105-09-01-01 & 24-09-2003 17:28 & $18: 28$ & 0.25 & $0.38-0.40$ & FB and Soft apex \\
\hline 53 & 80105-09-01-02 & 24-09-2003 19:17 & 19:53 & 0.30 & $0.36-0.40$ & FB and Soft apex \\
\hline 54 & 90022-09-03-00 & 15-04-2004 12:42 & $13: 50$ & 0.32 & $0.77-0.79$ & Upper HB \\
\hline
\end{tabular}


Table 2: Log of observations for which anti-correlated hard and soft X-ray lags were detected (first four columns). The cross-correlation coefficients (CCs) along with their delays are shown in the fifth and sixth columns. The last two columns show hardness ratios and the corresponding branch positions on the Z-track, respectively.

\begin{tabular}{|c|c|c|c|c|c|c|c|}
\hline No. & ObsID & Start Time & Stop Time & $\mathrm{CC}$ & Delay (s) & Hardness Ratio & Branch position on HID plane \\
\hline 1 & 20053-02-01-00 & $30-05-1997,09: 28$ & $17: 14$ & a) $-0.40 \pm 0.09$ & a) $34.05 \pm 18.02$ & $0.54-0.56$ & Upper NB \\
\hline 2 & 20053-02-01-01 & 25-07-1997, 21:41 & 01:07 & $-0.60 \pm 0.06$ & $-249 \pm 108$ & $0.67-0.74$ & Lower and Middle HB \\
\hline 3 & 20055-01-04-00 & 28-07-1997, 18:27 & $20: 24$ & $-0.72 \pm 0.05$ & $31.36 \pm 12.04$ & $0.52-0.59$ & Upper NB \\
\hline 5 & $30042-01-04-00$ & 08-10-1998, 8:18 & $10: 14$ & $-0.55 \pm 0.08$ & $209.18 \pm 20.22$ & $0.47-0.58$ & Lower and Upper NB \\
\hline \multirow[t]{2}{*}{6} & $30042-01-06-00$ & $26-10-1998,04: 58$ & 09:12 & a) $-0.41 \pm 0.04$ & a) $580 \pm 142$ & $0.39-0.44$ & Soft apex \\
\hline & & & & b) $-0.53 \pm 0.03$ & b) $748 \pm 62$ & $0.69-0.73$ & Upper HB \\
\hline 7 & 30042-01-09-00 & 04-11-1998, 05:01 & 08:15 & $-0.51 \pm 0.05$ & $150 \pm 21$ & $0.69-0.73$ & Upper HB \\
\hline 8 & $30042-01-11-00$ & 09-11-1998, 06:38 & $10: 45$ & a) $-0.49 \pm 0.08$ & a) $43 \pm 18$ & $0.61-0.65$ & Lower HB \\
\hline \multirow[t]{2}{*}{10} & $30042-01-15-00$ & 20-11-1998, 16:10 & $18: 34$ & a) $-0.59 \pm 0.05$ & a) $748 \pm 51$ & $0.58-0.66$ & Hard apex and Lower HB \\
\hline & & & & b) $-0.78 \pm 0.04$ & b) $58 \pm 8$ & $0.57-0.62$ & Hard apex \\
\hline 11 & $30042-01-16-000$ & 21-11-1998, 00:10 & 08:03 & $-0.57 \pm 0.04$ & $67 \pm 14$ & $0.38-0.40$ & FB \\
\hline 12 & $30042-01-16-00$ & 21-11-1998, 08:37 & $10: 47$ & $-0.59 \pm 0.02$ & $220 \pm 32$ & $0.37-0.42$ & FB and Soft apex \\
\hline \multirow[t]{2}{*}{13} & $30042-01-18-00$ & 21-11-1998, 22:35 & 05:14 & a) $-0.42 \pm 0.06$ & a) $-160 \pm 70$ & $0.58-0.61$ & Lower HB, Hard apex and Upper NB \\
\hline & & & & b) $-0.61 \pm 0.08$ & b) $65 \pm 20$ & $0.60-0.61$ & Lower HB \\
\hline 14 & $40018-02-02-030$ & 03-03-2000, 15:25 & $23: 24$ & $-0.51 \pm 0.03$ & $284 \pm 13$ & $0.40-0.52$ & Lower NB \\
\hline 15 & $40018-02-02-210$ & 04-03-2000, 17:04 & $00: 58$ & $-0.58 \pm 0.02$ & $-920 \pm 102$ & $0.38-0.50$ & FB and Lower NB \\
\hline 16 & 90022-09-01-00 & 02-04-2003, 02:59 & $02: 36$ & $-0.60 \pm 0.05$ & $-256 \pm 102$ & $0.57-0.60$ & Hard apex \\
\hline
\end{tabular}


Table 3: Best-fit spectral parameters for the ObsID 30042-01-13-00. The letters A, B, C, and D represent the segments in the light curve. The subscripts dbb and bb represent the disk black body

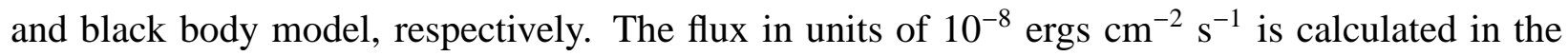
energy band 3-25 keV and otherwise it is mentioned. Errors are quoted at a $90 \%$ confidence level.

\begin{tabular}{|c|c|c|c|c|c|c|c|c|}
\hline \multirow[t]{2}{*}{ Parameters } & \multicolumn{2}{|c|}{ A } & \multicolumn{2}{|c|}{ B } & \multicolumn{2}{|c|}{$\mathrm{C}$} & \multicolumn{2}{|c|}{$\mathrm{D}$} \\
\hline & $\mathrm{A}_{d b b}$ & $\mathrm{~A}_{b b}$ & $\mathrm{~B}_{d b b}$ & $\mathrm{~B}_{b b}$ & $\mathrm{C}_{d b b}$ & $\mathrm{C}_{b b}$ & $\mathrm{D}_{d b b}$ & $\mathrm{D}_{b b}$ \\
\hline$k T_{\text {in }}(\mathrm{keV})^{a}$ & $2.10 \pm 0.07$ & - & $2.36 \pm 0.08$ & - & $2.82 \pm 0.03$ & - & $2.87 \pm 0.03$ & - \\
\hline$N_{\text {disk }} b$ & $52.52 \pm 5.50$ & - & $25.94 \pm 2.26$ & - & $16.54 \pm 1.80$ & - & $16.25 \pm 2.01$ & - \\
\hline$k T_{b b}(\mathrm{keV})^{c}$ & - & $1.51 \pm 0.10$ & - & $1.67 \pm 0.11$ & - & $1.96 \pm 0.05$ & - & $2.04 \pm 0.05$ \\
\hline$N_{b b}{ }^{d}$ & - & $0.09 \pm 0.01$ & - & $0.06 \pm 0.01$ & - & $0.07 \pm 0.02$ & - & $0.08 \pm 0.02$ \\
\hline $\mathrm{kT}_{e}^{e}(\mathrm{keV})$ & $3.3 \pm 0.2$ & $3.00 \pm 0.01$ & $3.3 \pm 0.3$ & $3.0 \pm 0.1$ & $6.1 \pm 1.3$ & $3.5 \pm 0.3$ & $12.8 \pm 2.3$ & $3.7 \pm 0.4$ \\
\hline$\tau^{f}$ & $8.7 \pm 0.6$ & $9.3 \pm 0.5$ & $8.6 \pm 0.8$ & $9.8 \pm 0.6$ & $4.3 \pm 0.7$ & $8.4 \pm 0.8$ & $2.2 \pm 1.2$ & $8.1 \pm 0.9$ \\
\hline disk flux & 1.54 & - & 1.22 & - & 1.60 & - & 1.72 & - \\
\hline CompTT flux & 2.27 & - & 2.20 & - & 1.19 & - & 1.00 & - \\
\hline BB flux ${ }^{g}$ & - & 6.14 & - & 4.34 & - & 5.89 & - & 6.19 \\
\hline CompTT flux & - & 3.06 & - & 3.07 & - & 2.26 & - & 2.14 \\
\hline Delay (s) & $202 \pm 92$ & - & - & - & $139 \pm 36$ & - & $72 \pm 25$ & - \\
\hline pivot energy (keV) & $6.7 \pm 0.1$ & - & - & - & $12.2 \pm 0.2$ & - & $15.6 \pm 0.3$ & - \\
\hline$v_{\text {centriod }}{ }^{h}(\mathrm{~Hz})$ & $51.7 \pm 1.8$ & - & $39.1 \pm 1.0$ & - & $24.2 \pm 0.2$ & - & $21.5 \pm 0.3$ & - \\
\hline$\chi^{2} /$ dof & $23 / 46$ & $25 / 46$ & $25 / 46$ & $23 / 46$ & $28 / 46$ & $27 / 46$ & $26 / 46$ & $27 / 46$ \\
\hline
\end{tabular}

${ }^{a}$ Inner disk temperature using the disk black body model.

${ }^{b}$ Normalization of the disk black body model.

${ }^{c}$ Temperature of the black body model.

${ }^{d}$ Normalization of the black body model.

${ }^{e}$ Electron temperature.

${ }^{f}$ Optical depth of the Compton cloud.

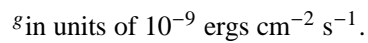

${ }^{h} \mathrm{HBO}$. 
Table 4: Best-fit spectral parameters for the spectra of A and B segments for ObsID 20053-02-0101. Errors are quoted at a $90 \%$ confidence level.

\begin{tabular}{ccc}
\hline \hline Parameters & \multicolumn{2}{c}{$20053-02-01-01$} \\
\hline \hline$k T_{\text {in }}(\mathrm{keV})_{-}^{a}$ & $2.84 \pm 0.01$ & $2.95 \pm 0.04$ \\
$N_{\text {disk }}^{b}$ & $16.7 \pm 0.3$ & $14.6 \pm 0.1$ \\
$\mathrm{kT}_{e-}^{c}(\mathrm{keV})$ & $7.7 \pm 0.2$ & $13.1 \pm 0.3$ \\
$\tau_{-}^{d}$ & $3.5 \pm 0.7$ & $2.0 \pm 0.5$ \\
Delay $_{-}(\mathrm{s})$ & $-249 \pm 108$ & - \\
$\mathrm{pivot} \mathrm{energy}(\mathrm{keV})$ & $8.9 \pm 0.1$ & - \\
$v_{\text {centriod_}}^{e}(\mathrm{~Hz})$ & $23.1 \pm 0.1$ & $18.2 \pm 0.1$ \\
$\mathrm{kHz}_{\text {centriod }}(\mathrm{Hz})$ & $588 \pm 28$ & $546 \pm 8$ \\
$\chi^{2} / \mathrm{dof}$ & $31 / 47$ & $36 / 47$ \\
\hline \hline
\end{tabular}

${ }^{a}$ Inner disk temperature using the disk black body model.

${ }^{b}$ Normalization of the disk black body model. ${ }^{c}$ Electron temperature.

${ }^{d}$ Optical depth of the Compton cloud. ${ }^{e} \mathrm{HBO}$. 


\section{REFERENCES}

Agrawal, V. K., \& Sreekumar, P. 2003, MNRAS, 346, 933

Agrawal, V. K., \& Misra, R. 2009, MNRAS, 398, 1352

Arnaud, K. A. 1996, in Jacoby G. H., Barnes J., eds, ASP Conf. Ser. 105, Astronomical Data Analysis Software and Systems V, San Francisco, p. 17

Asai, K., Dotani, T., Mitsuda, K. et al. 1994, PASJ, 46, 479

Balucinska-Church, M., Gibiec, A., Jackson, N. K., \& Church, M. J. 2010, A\&A, 512, 9

Belloni, T. 2010, The Jet Paradigm (Lecture Notes in Physics), Vol. 794 (Berlin: Springer)

Berendsen, S. G. H., Fender, R., Kuulkers, E., Heise, J., \& van der Klis, M. 2000, MNRAS, 318, 599

Bottcher, M., \& Liang, E. P. 1999, ApJ, 511, L37

Bradt, H., Naranan, S., Rappaport, S., \& Spada, G. 1968, ApJ, 152, 1005

Chakrabarti, S. K., \& Manickam, S. G. 2000, ApJL, 531, L41

Church, M. J., \& Balucinska-Church, M. 2001, A\&A, 369, 915

Choudhury, M., \& Rao, A. R. 2004, ApJL, 616, L143

Choudhury, M., Rao, A. R., Dasgupta, S., Pendharkar, J., Sriram, K., \& Agrawal, V. K. 2005, ApJ, 631,1072

Di Salvo, T., Stella, L., Robba, N. R., van der Klis, M., Burderi, L., Israel, G. L., Homan, J., Campana, S., Frontera, F., \& Parmar, A. N. 2000, ApJ, 544, L119

Di Salvo, T., Robba, N. R., Iaria, R. et al. 2001, ApJ, 554, 4 
Done, C., Zycki, P. T., \& Smith, D. A. 2002, MNRAS, 331, 453

Done, C., \& Gierlinski, M. 2006, MNRAS, 367, 659

Done, C., \& Kubota, A. 2006, MNRAS, 371, 1216

Done, C., Gierlinski, M., \& Kubota, A. 2007, A\&AR, 15, 1

Done, C., \& Diaz Trigo, M. 2010, MNRAS, 407, 2287

Fender, R. P. 1999, ESO workshop Black Holes in binaries and galactic nuclei, ed. L. Kaper, E. P. J. van den Heuvel, \& P. A. Woudt (Springer-Verlag) [arXiv:astro-ph/9911176]

Fender, R. P., \& Hendry, M. A. 2000, MNRAS, 317, 1

Fender, R. P., Homan, J., \& Belloni, T. M. 2009, MNRAS, 396, 1370

Fisher, P. C., Jordan, W. C., Meyerott, A. J., Acton, L. W., \& Roethig, D. T. 1968, ApJ, 151, 1

Frank, J., King, A. R., \& Raine, D. J. 2002, Accretion Power in Astrophysics, 3rd ed., Cambridge Univ. Press, Cambridge

Hasinger, G., Priedhorsky, W. C., \& Middleditch, J. 1989, ApJ, 337, 843

Hasinger, G., \& van der Klis, M. 1989, A\&A, 225, 79

Hasinger, G., van der Klis, M., Ebisawa, K., Dotani, T., \& Mitsuda, K. 1990, A\&A, 235, 131

Homan, J. et al. 2001, ApJS, 132, 377

Homan, J. et al. 2007, ApJ, 656, 420

Homan, J., van der Klis, M., Fridriksson, J. K. et al. 2010, ApJ, 719, 201

Ingram, A., \& Done, C. 2010, MNRAS, 405, 2447 
Jackson, N. K., Church, M. J., \& Balucinska-Church, M. 2009, A\&A, 494, 1059

Jahoda, K. et al. 2006, ApJS, 163, 401

Jonker, P. G., van der Klis, M., Wijnands, R. et al. 2000, ApJ, 537, 374

Jonker, P. G., van der Klis, M., Homan, J., Mendez, M., Lewin, W. H. G., Wijnands, R., \& Zhang, W. 2002, MNRAS, 333, 665

Kazanas, D., Hua, X. M., \& Titarchuk, L. 1997, ApJ, 480, 735

Kuulkers, E., van der Klis, M., Oosterbroek, T. et al. 1994, A\&A, 289, 759

Kuulkers, E., van der Klis, M., Oosterbroek, T., van Paradijs, J., \& Lewin, W. H. G. 1997, MNRAS, 287, 495

Lei, Y. J., Qu, J. L., Song, L. M., Zhang, C. M., Zhang, S., Zhang, F., Wang, J. M., Li, Z. B., \& Zhang, G. B. 2008, ApJ, 677, 461

Li, S. L., Xue, L., \& Lu, J. F. 2007, ApJ, 666, 368

Lin, D., Remillard, R. A., \& Homan, J. 2007, ApJ, 667, 1073

Lin, D., Remillard, R. A., \& Homan, J. 2009, ApJ, 696, 1257

Lewin, W. H. G., Lubin, L. M., Tan, J. et al. 1992, MNRAS 256, 545

Meyer, F., Liu, B. F., \& Meyer-Hofmeister, E. 2007, A\&A, 463, 1

Miller, J. M., Homan, J., Steeghs, D., Rupen, M., Hunstead, R. W., Wijnands, R., Charles, P. A., \& Fabian, A. C. 2006, ApJ, 653, 525

Migliari, S., \& Fender, R. 2006, MNRAS, 366, 79

Migliari, S. et al. 2007, ApJ, 671, 706 
Mitsuda, K., Inoue, H., Koyama, K., Makishima, K., Matsuoka, M., Ogawara, Y., Suzuki, K., Tanaka, Y., Shibazaki, N., \& Hirano, T. 1984, PASJ, 36, 741

Ng, C., Diaz Trigo, M., Cadolle Bel, M., \& Migliari, S. 2010, A\&A, 522, 96

Nobili, L., Turolla, R., Zampieri, L., \& Belloni, T. 2000, ApJ, 538, L137

Nowak, M. A., Wilms, J., \& Vaughan, B. A. 1999, ApJ, 515, 726

O’Brien, K. et al. 2004, MNRAS, 350, 587

Paizis, A., Ebisawa, K., Tikkanen, T., Rodriguez, J., Chenevez, J., Kuulkers, E., Vilhu, O., \& Courvoisier, T. J. L. 2005, A\&A, 444, 357

Paizis, A. et al. 2006, A\&A, 459, 187

Penninx, W. 1989, in Hunt J., Battrick B., eds, Proc. of the 23rd ESLAB symposium on Two Topics in X-ray Astronomy, Bologna, ESA SP-296, p. 185

Psaltis, D., Lamb, F. K., \& Miller, G. S. 1995, ApJ, 454, L137

Qu, J. L., Chen, Y., Wu, M., Chen, L., \& Song, L. M. 2004, Ap\&SS, 293, 441

Remillard, R. A., \& McClintock, J. E. 2006, ARA\&A, 44, 49

Rykoff, E. S., Miller, J. M., Steeghs, D., \& Torres, M. A. P. 2007, ApJ, 666, 1129

Sriram, K., Agrawal, V. K., Pendharkar Jayant, K., \& Rao, A. R. 2007, ApJ, 661, 1055

Sriram, K., Agrawal, V. K., \& Rao, A. R. 2009, RAA, 9, 901

Sriram, K., Rao, A. R., \& Choi, C. S. 2010, ApJ, 725, 1317

Sriram, K., Choi, C. S., \& Rao, A. R. 2011a, A\&A, 525, A146

Sriram, K., Rao, A. R., \& Choi, C. S. 2011b, ApJL, 743, L31 
Sturner, S. J., \& Shrader, C. R. 2005, ApJ, 625, 923

Tan, J. et al. 1992, ApJ, 385, 314

Timmer, J. \& Koenig, M. 1995, A\&A, 300, 707

Titarchuk, L. 1994, ApJ, 434, 570

Titarchuk, L., \& Fiorito, R. 2004, ApJ, 612, 988

Titarchuk, L., \& Shaposhnikov, N. 2005, ApJ, 626, 298

Uttley, P., Wilkinson, T., Cassatella, P., Wilms, J., Pottschmidt, K., Hanke, M., \& Bock, M. 2011, MNRAS, Letters, 413: no. doi: 10.1111/j.1745-3933.2011.01056.x

van der Klis, M., Jansen, F., van Paradijs, J. et al. 1985, Nature, 316, 225

van der Klis, M., Stella, L., White, N. E., Jansen, F., \& Parmar, A. N. 1987, ApJ, 316, 411

van der Klis, M. 2006, in Lewin W., van der Klis M., eds, Compact Stellar X-ray Sources, Cambridge Univ. Press, Cambridge, p. 39

Vaughan, B. A., van der Klis, M., Lewin, W. H. G., van Paradijs, J., Mitsuda, K., \& Dotnai, T. 1999, A\&A, 343, 197

White, N. E., Peacock, A., Hasinger, G., Mason, K. O., Manzo, G., Taylor, B. G., \& Branduardi-Raymont, G. 1986, MNRAS, 218, 129

Wijnands, R., Mendez, M., van der Klis, M., Psaltis, D., Kuulkers, E., \& Lamb, F. K. 1998, ApJ, 504, L35

Zdziarski, A. A., Johnson, W. N., \& Magdziarz, P. 1996, MNRAS, 283, 193

Zdziarski, A. A., Poutanen, J., Paciesas, W. S., \& Linqing, W. 2002, ApJ, 578, 357 
This manuscript was prepared with the AAS LATEX macros v5.2. 